\title{
AN ITERATIVE PROCEDURE TO SOLVE A COUPLED TWO-FLUIDS TURBULENCE MODEL*
}

\author{
Tomas Chacón Rebollo ${ }^{1}$, Stéphane Del Pino ${ }^{2}$ And Driss Yakoubi ${ }^{3}$
}

\begin{abstract}
This paper introduces a scheme for the numerical approximation of a model for two turbulent flows with coupling at an interface. We consider the variational formulation of the coupled model, where the turbulent kinetic energy equation is formulated by transposition. We prove the convergence of the approximation to this formulation for 3D flows for large turbulent viscosities and smooth enough flows, whenever bounded in $W^{1, p}$ Sobolev norms for $p$ large enough. Under the same assumptions, we show that the limit is a solution of the initial problem. Finally, we give some numerical experiments to enlighten the theoretical work.
\end{abstract}

Mathematics Subject Classification. 63N30, 76M10.

Received March 29, 2008. Revised August 1st, 2009.

Published online February 23, 2010.

\section{INTRODUCTION}

In this contribution we focus our attention on the modelling of the surface layer between the atmosphere and the ocean. We are interested in designing effective procedures to solve the following coupled model:

$$
\left\{\begin{aligned}
-\nabla \cdot\left(\alpha_{i}\left(k_{i}\right) \nabla \mathbf{u}_{i}\right)+\operatorname{grad} p_{i} & =\mathbf{f}_{i} \text { in } \Omega_{i}, \\
\nabla \cdot \mathbf{u}_{i} & =0 \text { in } \Omega_{i}, \\
-\nabla \cdot\left(\gamma_{i}\left(k_{i}\right) \nabla k_{i}\right) & =\alpha_{i}\left(k_{i}\right)\left|\nabla \mathbf{u}_{i}\right|^{2} \text { in } \Omega_{i}, \\
\mathbf{u}_{i} & =\mathbf{0} \text { on } \Gamma_{i}, \\
k_{i} & =0 \text { on } \Gamma_{i}, \\
\alpha_{i}\left(k_{i}\right) \partial_{\mathbf{n}_{i}} \mathbf{u}_{i}-p_{i} \mathbf{n}_{i}+\kappa\left(\mathbf{u}_{i}-\mathbf{u}_{j}\right)\left|\mathbf{u}_{i}-\mathbf{u}_{j}\right| & =\mathbf{0} \text { on } \Gamma, 1 \leq i \neq j \leq 2, \\
k_{i} & =\lambda\left|\mathbf{u}_{1}-\mathbf{u}_{2}\right|^{2} \text { on } \Gamma .
\end{aligned}\right.
$$

Where each triple $\left(\mathbf{u}_{i}, p_{i}, k_{i}\right)$ is defined in the domain $\Omega_{i}, 1 \geq i \geq 2$. The generic point in $\mathbb{R}^{2}$, resp. in $\mathbb{R}^{3}$, is denoted by $\mathbf{x}=(x, z)$, resp. $\mathbf{x}=(x, y, z)$.

\footnotetext{
Keywords and phrases. Ocean-atmosphere coupling, turbulent flows, convergence analysis, iterative method, spectral method.

* T. Chacón Rebollo was partially funded by EU Marie-Curie Fellowship Programme, by Spanish Government Grant MTM200601275 and by Junta de Andalucía Grant P07-FQM-02538.

${ }^{1}$ Departamento de Ecuaciones Diferenciales y Analisis Numerico, Universidad de Sevilla, Spain.

2 CEA, DAM, DIF, 91297 Arpajon, France.

${ }^{3}$ Laboratoire Jacques-Louis Lions, Université Pierre et Marie Curie, 4 place Jussieu, 75005 Paris Cedex, France.
} 
System (1.1) is a simplified model for two stationary turbulent flows in adjacent domains, coupled by boundary conditions on the interface, such as the system atmosphere-ocean. Indeed, it is a simplified mathematical formulation of the RANS (Reynolds Averaged Navier-Stokes) model of order 1 used to simulate a stationary mean flow when convection is neglected. This kind of modelling is often used in engineering or geophysics, see for instance Bernardi et al. [6], Launder and Spalding [16], Mohammadi and Pironneau [21], Piquet [22], Wilcox [23].

In what follows, $\Omega_{i}(i=1,2)$ are bounded domains of $\mathbb{R}^{d}, d=2,3$, which are either convex or of class $\mathbb{C}^{1,1}$, with boundaries $\partial \Omega_{i}=\Gamma_{i} \cup \Gamma, \Gamma=\bar{\Omega}_{1} \cap \bar{\Omega}_{1}$ being the interface between the two fluids. $\Gamma$ is assumed to be flat. Indeed, we assume that the so-called "rigid lid hypothesis" (introduced by Bryan in [9]) holds, an hypothesis which is standard in geophysics and oceanography. Each of the two turbulent fluids is modeled by a simplified one-equation turbulence model whose unknowns are the velocity $\mathbf{u}_{i}$ and the turbulent kinetic energy (TKE) $k_{i}$.

In the first equation we model the generation of eddy viscosity in flow $i$ by the term

$$
-\alpha_{i}\left(k_{i}\right) \nabla \mathbf{u}_{i} .
$$

The (positive) quantity $\alpha_{i}\left(k_{i}\right)$ is the eddy viscosity. This is a simplification of the usual modelling of Reynolds Stress Tensor by

$$
R_{i} \simeq-\alpha_{i}\left(k_{i}\right)\left(\nabla \mathbf{u}_{i}+\nabla^{t} \mathbf{u}_{i}\right)
$$

We prefer the first expression for simplicity of mathematical analysis, although our analysis still holds for the second one. We also neglect transport effects, we intend to analyze them in a forthcoming paper. The fluids are assumed to be incompressible (second equation). In the third equation we model the generation of TKE by means of a production source term

although the physical one should be

$$
\alpha_{i}\left(k_{i}\right)\left|\nabla \mathbf{u}_{i}\right|^{2}
$$

$$
\alpha_{i}\left(k_{i}\right)\left|\nabla \mathbf{u}_{i}+\nabla^{t} \mathbf{u}_{i}\right|^{2} .
$$

Again, we prefer the first expression for simplicity of mathematical treatment. Also the turbulent diffusion of TKE is the function $\gamma_{i}\left(k_{i}\right)$. We neglect the viscous dissipation effects, to avoid to manage an additional statistic of the turbulence (a mixing length or the turbulent dissipation $\varepsilon$, for instance). We assume nonslipping boundary conditions in the boundary parts $\Gamma_{i}$ for simplicity (fourth and fifth equations). These in practice are replaced by wall-laws to simulate the generation of turbulence on solid boundaries. The sixth equation globally models the interaction of the two boundary layers on one and another side of the interface $\Gamma$ as friction effects, by means of a set of boundary conditions similar to Manning's law. Finally, the last equation models the production of TKE in the interface. The coefficients $\kappa_{i}$ and $\lambda$ are positive.

We assume that the turbulent diffusions $\alpha_{i}$ and $\gamma_{i}$ belong to $W^{1, \infty}(\mathbb{R})$ and verify $\alpha_{i} \geq \nu, \gamma_{i} \geq \nu$, for some $\nu>0$. The eddy diffusions usually are unbounded functions of the TKE of the form $a+b \sqrt{k}$, as we use in the numerical simulations reported in Section 5 (see for instance $[8,17,21]$ ). But this renders the analysis much more complex even for a one-fluid turbulence model (see [18]). So we consider a simplified model, that still includes several realistic non-linear interactions.

System (1.1) was studied in [3] where existence and uniqueness of small smooth solutions were proved. Spectral and Finite Element discretizations were studied in subsequent papers by the same authors and coworkers (see [4,5]). In these papers, the ability of these discretization techniques to approach the solution of model (1.1) was proved. However, in both cases the discretizations achieved consisted in fully non-linear sets of algebraic equations. Our purpose here is to derive iterative procedures to solve system (1.1) that decouples the interaction of the problem, leading to mildly non-linear problems.

Let us introduce the function spaces

$$
\begin{aligned}
\mathbf{X}_{i} & =\left\{\mathbf{v}_{i} \in \mathbf{H}^{1}\left(\Omega_{i}\right) ; \quad \mathbf{v}_{i}=\mathbf{0} \quad \text { on } \Gamma_{i}\right\}, \\
L_{0}^{2}\left(\Omega_{i}\right) & =\left\{q_{i} \in L^{2}\left(\Omega_{i}\right) ; \quad \int_{\Omega_{i}} q_{i}=0\right\} .
\end{aligned}
$$


Consider also two conjugate positive real numbers $r$ and $r^{\prime}$ i.e.

$$
\frac{1}{r}+\frac{1}{r^{\prime}}=1, \quad \text { such that } r>d .
$$

We introduce the following iterative procedure to solve (1.1): once known $\mathbf{u}_{i}^{n} \in \mathbf{X}_{i}, p_{i}^{n} \in L_{0}^{2}\left(\Omega_{i}\right), k_{i} \in W^{1, r^{\prime}}\left(\Omega_{i}\right)$, $i=1,2$, solve:

Problem 1. Obtain $\mathbf{u}_{i}^{n+1} \in \mathbf{X}_{i}, i=1,2$, such that

$$
\begin{gathered}
\int_{\Omega_{i}} \alpha_{i}\left(k_{i}^{n}\right) \nabla \mathbf{u}_{i}^{n+1}: \nabla \mathbf{v}_{i} \mathrm{~d} \mathbf{x}-\int_{\Omega_{i}}\left(\nabla \cdot \mathbf{v}_{i}\right) p_{i}^{n+1} \mathrm{~d} \mathbf{x}+\kappa_{i} \int_{\Gamma}\left|\mathbf{u}_{i}^{n+1}-\mathbf{u}_{j}^{n+1}\right|\left(\mathbf{u}_{i}^{n+1}-\mathbf{u}_{j}^{n+1}\right) \cdot \mathbf{v}_{i} \mathrm{~d} \tau=\int_{\substack{\Omega_{i} \\
\forall \mathbf{v}_{i} \in \mathbf{X}_{i},}} \mathbf{f}_{i} \cdot \mathbf{v}_{i} \mathrm{~d} \tau, \\
\int_{\Omega_{i}}\left(\nabla \cdot \mathbf{u}_{i}^{n+1}\right) q_{i} \mathrm{~d} \mathbf{x}=0, \quad \forall q_{i} \in L_{0}^{2}\left(\Omega_{i}\right),
\end{gathered}
$$

and

Problem 2. Obtain $k_{i}^{n+1} \in W^{1, r^{\prime}}\left(\Omega_{i}\right), i=1,2$, such that

$$
\begin{aligned}
& k_{i}^{n+1}=0 \quad \text { on } \Gamma_{i}, \quad k_{i}^{n+1}=\lambda\left|\mathbf{u}_{1}^{n+1}-\mathbf{u}_{2}^{n+1}\right|^{2} \quad \text { on } \Gamma, \\
& \int_{\Omega_{i}} \gamma_{i}\left(k_{i}^{n}\right) k_{i}^{n+1} \varphi_{i} \mathrm{~d} \mathbf{x}=\int_{\Omega_{i}} \alpha_{i}\left(k_{i}^{n}\right)\left|\nabla \mathbf{u}_{i}^{n+1}\right|^{2} \varphi_{i} \mathrm{~d} \mathbf{x}, \quad \forall \varphi_{i} \in W_{0}^{1, r}\left(\Omega_{i}\right) .
\end{aligned}
$$

Remark 1.1. We take $r>d$ to give a sense to the equation for the $k_{i}$ in model (1.1). Indeed, the term $\alpha_{i}\left(k_{i}^{n}\right)\left|\nabla \mathbf{u}_{i}^{n+1}\right|^{2}$ belongs to $L^{1}\left(\Omega_{i}\right)$, and it follows from the Sobolev Imbedding Theorem that the test function $\varphi_{i}$ in Problem 2 belongs to $L^{\infty}\left(\Omega_{i}\right)$, so that the right-hand member is well defined.

Observe that Problem 1 is in reality non-linear due to the presence of the Manning-like source term. This is a mild non-linearity due to the monotonic nature of this term, that may be made explicit in practice if mass-lumping techniques are used.

Our main result states that if the sequences $\left(\mathbf{u}_{i}^{n}\right)_{n}$ and $\left(k_{i}^{n}\right)_{n}$ are respectively bounded in $W^{1,3+\varepsilon}\left(\Omega_{i}\right)^{d}$ and $W^{1,3}\left(\Omega_{i}\right)$, then, for small enough data (in a convenient sense), the iterative scheme is contracting. This regularity is realistic, as it is not far from the $W^{1,2}$ regularity that has been proved for problem (1.1) for general data. The main ingredients to show the convergence of our scheme are the convenient choices of test functions, and the use of the harmonic liftings $R_{i}$ of Dirichlet boundary conditions on $\Gamma$ on the $\Omega_{i}$ (see the proof of Thm. 3.4).

Our paper is organised as follows. In Section 2 we introduce a weak formulation of the above iterative procedure. Section 3 is devoted to prove the contractiveness of the TKE sequence. Due to the production term of the TKE on interface $\Gamma: k_{i}=\lambda\left|\mathbf{u}_{1}-\mathbf{u}_{2}\right|^{2}$, it is necessary to estimate the expression

$$
||\left|\mathbf{u}_{1}^{n+1}-\mathbf{u}_{2}^{n+1}\right|^{2}-\left.\left|\mathbf{u}_{1}^{n}-\mathbf{u}_{2}^{n}\right|^{2}\right|_{H_{00}^{\frac{1}{2}(\Gamma)}},
$$

where the special space $H_{00}^{\frac{1}{2}}(\Gamma)$ is the subspace of $H^{\frac{1}{2}}(\Gamma)$ whose extension by zero to $\partial \Omega_{1}$ (for instance, it could be also to $\left.\partial \Omega_{2}\right)$ belongs to $H^{\frac{1}{2}}\left(\partial \Omega_{1}\right)$. An intrinsic scalar product on $H_{00}^{\frac{1}{2}}(\Gamma)$ is defined as

$$
((u, v))_{H_{00}^{\frac{1}{2}}(\Gamma)}=\int_{\Gamma} u(x) v(x) \mathrm{d} x+\int_{\Gamma} \int_{\Gamma} \frac{(u(x)-u(y))(v(x)-v(y))}{|x-y|^{d}} \mathrm{~d} x \mathrm{~d} y+\int_{\Gamma} \frac{u(x) v(x)}{d(x, \partial \Gamma)},
$$

where the first two summands define the $H^{\frac{1}{2}}(\Gamma)$ scalar product (see Adams and Fournier [1], Thm. 7.48). Its expression involves the distance $d(x, \partial \Gamma)$ to the boundary of $\partial \Gamma$. It comes from the restriction to $H_{00}^{\frac{1}{2}}(\Gamma)$ of the scalar product in $H^{\frac{1}{2}}\left(\partial \Omega_{1}\right)$ for instance. It is given by Lions and Magenes in [20], Chapter 1, Theorem 11.7. 
The estimation of (1.3) is done using a Grisvard's result (Lem. 3.5 in the paper at hand, see [14] for the original reference) on estimates in $W^{s, p}$ of products of functions of $W^{s_{j}, p_{j}}$, for some real numbers $s, s_{j}$ and some non-negative integers $p, p_{j}, j=1,2$. Concerning the sequence of pressure iterates $\left(p_{i}^{n}\right)_{n}$, we use a specific inf-sup condition (see Cor. 3.7) to show that it is a Cauchy sequence, see Theorem 3.8.

The convergence analysis is performed in Section 4. In Theorem 4.1 we prove that the triple $\left(\mathbf{u}_{i}^{n}, k_{i}^{n}, p_{i}^{n}\right)_{n}$ has a unique limit, which is a solution of the variational formulation (2.3)-(2.4).

We finally present some numerical tests in Section 5. These tests are realized with the software FreeFEM3D (see [12]) in meaningful situations, that agree with the expectations of our result.

\section{ItERATIVE SCHEME}

We shall at first describe the weak formulation of problem (1.1). We assume that $\alpha_{i}$ and $\gamma_{i}$ are bounded functions from the set of nonnegative real numbers $\mathbb{R}_{+}$onto $\mathbb{R}$, and belong to $W^{1, \infty}\left(\Omega_{i}\right)$, which satisfy

$$
\forall \ell \in \mathbb{R}_{+}, \quad \delta_{1} \geq \alpha_{i}(\ell) \geq \nu \quad \text { and } \quad \delta_{1} \geq \gamma_{i}(\ell) \geq \nu,
$$

and

$$
\forall \ell \in \mathbb{R}_{+}, \quad\left|\alpha_{i}^{\prime}(\ell)\right| \leq \delta_{2} \quad \text { and } \quad\left|\gamma_{i}^{\prime}(\ell)\right| \leq \delta_{2},
$$

where $\delta_{1}, \delta_{2}$ and $\nu$ are positive constants.

System (1.1) admits the following variational formulation:

Find $\left(\mathbf{u}_{i}, p_{i}, k_{i}\right) \in \mathbf{X}_{i} \times L^{2}\left(\Omega_{i}\right) \times W^{1, r^{\prime}}\left(\Omega_{i}\right)$ such that, for all $\left(\mathbf{v}_{i}, q_{i}, \varphi_{i}\right) \in \mathbf{X}_{i} \times L^{2}\left(\Omega_{i}\right) \times W_{0}^{1, r}\left(\Omega_{i}\right)$,

$$
\begin{aligned}
a_{i}\left(k_{i} ; \mathbf{u}_{i}, \mathbf{v}_{i}\right)+b_{i}\left(\mathbf{v}_{i}, p_{i}\right)+\kappa_{i} \int_{\Gamma}\left|\mathbf{u}_{i}-\mathbf{u}_{j}\right|\left(\mathbf{u}_{i}-\mathbf{u}_{j}\right) \cdot \mathbf{v}_{i} \mathrm{~d} \tau & =\int_{\Omega_{i}} \mathbf{f}_{i} \cdot \mathbf{v}_{i} \mathrm{~d} \mathbf{x} \\
b_{i}\left(\mathbf{u}_{i}, q_{i}\right) & =0,
\end{aligned}
$$

and,

$$
\begin{array}{r}
k_{i}=0 \text { on } \Gamma_{i}, \quad k_{i}=\lambda\left|\mathbf{u}_{i}-\mathbf{u}_{j}\right|^{2} \quad \text { on } \Gamma, \quad \text { and } \\
C_{i}\left(k_{i} ; k_{i}, \varphi_{i}\right)=\int_{\Omega_{i}} \alpha_{i}\left(k_{i}\right)\left|\nabla \mathbf{u}_{i}\right|^{2} \varphi_{i} \mathrm{~d} \mathbf{x},
\end{array}
$$

where the forms $a_{i}(\cdot ; \cdot, \cdot), b_{i}(\cdot, \cdot)$ and $C_{i}(\cdot ; \cdot, \cdot)$ are defined by

$$
\begin{aligned}
a_{i}\left(\ell_{i} ; \mathbf{u}_{i}, \mathbf{v}_{i}\right) & =\int_{\Omega_{i}} \alpha\left(\ell_{i}\right) \nabla \mathbf{u}_{i}: \nabla \mathbf{v}_{i} \mathrm{~d} \mathbf{x}, \\
b_{i}\left(\mathbf{v}_{i}, q_{i}\right) & =-\int_{\Omega_{i}} q_{i} \nabla \cdot \mathbf{v}_{i} \mathrm{~d} \mathbf{x}, \\
C_{i}\left(\ell_{i} ; k_{i}, \varphi_{)}\right. & =\int_{\Omega_{i}} \gamma_{i}\left(\ell_{i}\right) \nabla k_{i} \cdot \nabla \varphi_{i} \mathrm{~d} \mathbf{x} .
\end{aligned}
$$

Note that the bilinear forms $a_{i}$ and $C_{i}$ in (2.3) depend on $k_{i}$.

Remark 2.1. Since $\mathbf{u}_{i} \in \mathbf{X}_{i}$ then its trace on $\Gamma$ belongs to $\mathbf{H}_{00}^{\frac{1}{2}}(\Gamma)$. Thus by using definition of this space $\left.\mathbf{u}_{i}\right|_{\Gamma}$ belongs to $\mathbf{H}^{\frac{1}{2}}(\Gamma)$ and applying the Sobolev embedding from $\mathbf{H}^{\frac{1}{2}}(\Gamma)$ into $L^{3}(\Gamma)^{\mathrm{d}}$, we conclude that the integral $\int_{\Gamma}\left|\mathbf{u}_{i}-\mathbf{u}_{j}\right|\left(\mathbf{u}_{i}-\mathbf{u}_{j}\right) \cdot \mathbf{v}_{i} \mathrm{~d} \tau$ is well defined. 
This formulation makes sense, as $\alpha_{i}\left(k_{i}\right)\left|\nabla \mathbf{u}_{i}\right|^{2} \in W^{1, r^{\prime}}\left(\Omega_{i}\right)$ when $\mathbf{u}_{i} \in \mathbf{X}_{i}$. In Lewandowski [19] it is proved that this formulation admits at least a solution.

We shall consider the following iterative procedure:

Given $\left(\mathbf{u}_{i}^{n}, p_{i}^{n}, k_{i}^{n}\right) \in \mathbf{X}_{i} \times L^{2}\left(\Omega_{i}\right) \times W^{1, r^{\prime}}\left(\Omega_{i}\right), i=1,2$, obtain $\left(\mathbf{u}_{i}^{n+1}, p_{i}^{n+1}, k_{i}^{n+1}\right) \in \mathbf{X}_{i} \times L^{2}\left(\Omega_{i}\right) \times W^{1, r^{\prime}}\left(\Omega_{i}\right)$, such that $\forall\left(\mathbf{v}_{i}, q_{i}, \varphi_{i}\right) \in \mathbf{X}_{i} \times L^{2}\left(\Omega_{i}\right) \times W_{0}^{1, r}\left(\Omega_{i}\right)$,

$$
\begin{aligned}
a_{i}\left(k_{i}^{n} ; \mathbf{u}_{i}^{n+1}, \nabla \mathbf{v}_{i}\right)+b_{i}\left(\mathbf{v}_{i}, p_{i}^{n+1}\right)+\kappa_{i} \int_{\Gamma}\left|\mathbf{u}_{i}^{n+1}-\mathbf{u}_{j}^{n+1}\right|\left(\mathbf{u}_{i}^{n+1}-\mathbf{u}_{j}^{n+1}\right) \cdot \mathbf{v}_{i} \mathrm{~d} \tau & =\int_{\Omega_{i}} \mathbf{f}_{i} \cdot \mathbf{v}_{i} \mathrm{~d} \tau, \\
\text { and } \quad b_{i}\left(\mathbf{u}_{i}^{n+1}, q_{i}\right) & =0,
\end{aligned}
$$

and

$$
\begin{aligned}
k_{i}^{n+1} & =0 \text { on } \Gamma_{i}, \\
k_{i}^{n+1} & =\lambda\left|\mathbf{u}_{1}^{n+1}-\mathbf{u}_{2}^{n+1}\right|^{2} \text { on } \Gamma, \\
C_{i}\left(k_{i}^{n} ; k_{i}^{n+1}, \varphi_{i}\right) & =\int_{\Omega_{i}} \alpha_{i}\left(k_{i}^{n}\right)\left|\nabla \mathbf{u}_{i}^{n+1}\right|^{2} \varphi_{i} \mathrm{~d} \mathbf{x} .
\end{aligned}
$$

\section{Contractiveness}

In this section we prove that the sequence of TKE $\left(k_{i}^{n}\right)_{n}$ is contracting, and that consequently the sequences of velocities $\left(\mathbf{u}_{i}^{n}\right)_{n}$ also is contracting, in the sense that

$$
\left\{\begin{array}{c}
\sum_{i=1}^{2}\left\|\nabla\left(\mathbf{u}_{i}^{n+1}-\mathbf{u}_{i}^{n}\right)\right\|_{L^{2}\left(\Omega_{i}\right)}^{2} \leq K \sum_{i=1}^{2}\left\|\nabla\left(k_{i}^{n}-k_{i}^{n-1}\right)\right\|_{L^{2}\left(\Omega_{i}\right)}^{2}, \quad \text { and } \\
\sum_{i=1}^{2}\left\|\nabla\left(k_{i}^{n+1}-k_{i}^{n}\right)\right\|_{L^{2}\left(\Omega_{i}\right)}^{2} \leq K \sum_{i=1}^{2}\left\|\nabla\left(k_{i}^{n}-k_{i}^{n-1}\right)\right\|_{L^{2}\left(\Omega_{i}\right)}^{2} .
\end{array}\right.
$$

We may interpret these inequalities in the sense that the sequence of pairs $\left(\mathbf{u}_{i}^{n}, k_{i}^{n}\right)_{n}$ is contracting in the Hilbert space $\mathbf{X}_{i} \times L^{2}\left(\Omega_{i}\right)$. However, to simplify our derivation, we shall not explicitly use this space. Finally, we show that the pressures $\left(p_{i}^{n}\right)_{n}$ is a Cauchy sequence.

We suppose from now on that the sequences $\left(\mathbf{u}_{i}^{n}\right)_{n}$ and $\left(k_{i}^{n}\right)_{n}$ verify the following hypothesis.

Hypothesis 3.1. $\forall n \in \mathbb{N}, \mathbf{u}_{i}^{n} \in W^{1,3+\varepsilon}\left(\Omega_{i}\right)^{d}$ and $k_{i}^{n} \in W^{1,3}\left(\Omega_{i}\right)$, and one has

$$
\left\|\mathbf{u}_{i}^{n}\right\|_{W^{1,3+\varepsilon}\left(\Omega_{i}\right)^{d}} \leq M, \quad\left\|k_{i}^{n}\right\|_{W^{1,3}\left(\Omega_{i}\right)} \leq M,
$$

where $M$ and $\varepsilon$ are two fixed positive numbers.

Remark 3.2. Note that the natural estimates for velocities in model (1.1) are in $H^{1}$ norm, not in $W^{1,3}$ norm. Indeed, choosing $\mathbf{v}_{i}$ equal to $\frac{1}{\kappa_{i}} \mathbf{u}_{i}^{n+1} \in \mathbf{X}_{i}$ in equation (2.5), and summing upon $i=1,2$ gives

$$
\sum_{i=1}^{2} \frac{1}{\kappa_{i}}\left(\int_{\Omega_{i}} \alpha_{i}\left(k_{i}^{n}\right)\left|\nabla \mathbf{u}_{i}^{n+1}\right|^{2} \mathrm{~d} x\right)+\int_{\Gamma}\left|\mathbf{u}_{1}^{n+1}-\mathbf{u}_{2}^{n+1}\right|^{3} \mathrm{~d} \tau=\sum_{i=1}^{2} \frac{1}{\kappa_{i}} \int_{\Omega_{i}} \mathbf{f}_{i} \mathbf{u}_{i}^{n+1} \mathrm{~d} x .
$$

Since the integrated term on $\Gamma$ is nonnegative and thanks to (2.1), we deduce

$$
\frac{\nu}{c_{M}} \sum_{i=1}^{2}\left\|\nabla \mathbf{u}_{i}^{n+1}\right\|_{L^{2}\left(\Omega_{i}\right)^{d}}^{2} \leq \frac{1}{c_{m}} \sum_{i=1}^{2} \int_{\Omega_{i}} \mathbf{f}_{i} \mathbf{u}_{i}^{n+1} \mathrm{~d} x .
$$


Using the Cauchy-Schwarz and Poincaré-Friedrichs inequalities, we obtain

$$
\sum_{i=1}^{2}\left\|\nabla \mathbf{u}_{i}^{n+1}\right\|_{L^{2}\left(\Omega_{i}\right)^{d}}^{2} \leq \frac{c}{\nu^{2}} \sum_{i=1}^{2}\left\|\mathbf{f}_{i}\right\|_{L^{2}\left(\Omega_{i}\right)^{d}}^{2}
$$

where $c$ is a positive constant, depending only on the domains $\Omega_{i}$ and the friction coefficients $\kappa_{i}$.

We next prove that the contractiveness of the TKE implies that of the velocities.

Lemma 3.3. Assume that Hypothesis 3.1 holds and that $\mathbf{f}_{i} \in L^{2}\left(\Omega_{i}\right)^{d}, i=1,2$. Then there exists a positive constant $c$, depending only on $\Omega_{i}$, such that

$$
\sum_{i=1}^{2}\left\|\nabla\left(\mathbf{u}_{i}^{n+1}-\mathbf{u}_{i}^{n}\right)\right\|_{L^{2}\left(\Omega_{i}\right)^{d}}^{2} \leq \frac{c \delta_{2}^{2} M^{2}}{\nu^{2}} \sum_{i=1}^{2}\left\|\nabla\left(k_{i}^{n}-k_{i}^{n-1}\right)\right\|_{L^{2}\left(\Omega_{i}\right)^{d}}^{2} .
$$

Proof. Let us take $\mathbf{v}_{i}=\frac{1}{\kappa_{i}}\left(\mathbf{u}_{i}^{n+1}-\mathbf{u}_{i}^{n}\right) \in \mathbf{X}_{i}$ as a test function in (2.5) at iterations $n$ and $n+1$. Then, calculating the difference between both obtained equations, and summing on $i=1,2$, yields

$$
\begin{aligned}
\sum_{i=1}^{2} \frac{1}{\kappa_{i}} \int_{\Omega_{i}} \alpha_{i}\left(k^{n}\right) \nabla\left(\mathbf{u}_{i}^{n+1}-\mathbf{u}_{i}^{n}\right): \nabla\left(\mathbf{u}_{i}^{n+1}-\mathbf{u}_{i}^{n}\right) \mathrm{d} \mathbf{x} & \\
& +\sum_{i=1}^{2} \frac{1}{\kappa_{i}} \int_{\Omega_{i}}\left(\alpha_{i}\left(k^{n}\right)-\alpha_{i}\left(k_{i}^{n-1}\right)\right) \nabla \mathbf{u}_{i}^{n}: \nabla\left(\mathbf{u}_{i}^{n+1}-\mathbf{u}_{i}^{n}\right) \mathrm{d} \mathbf{x} \\
& +\int_{\Gamma}\left(\left|\mathbf{u}_{1}^{n+1}-\mathbf{u}_{2}^{n+1}\right|\left(\mathbf{u}_{1}^{n+1}-\mathbf{u}_{2}^{n+1}\right)-\left|\mathbf{u}_{1}^{n}-\mathbf{u}_{2}^{n}\right|\left(\mathbf{u}_{1}^{n}-\mathbf{u}_{2}^{n}\right)\right) \cdot\left(\left(\mathbf{u}_{1}^{n+1}-\mathbf{u}_{2}^{n+1}\right)-\left(\mathbf{u}_{1}^{n}-\mathbf{u}_{2}^{n}\right)\right) \mathrm{d} \tau=0 .
\end{aligned}
$$

The following inequality holds for all vectors $\mathbf{a}, \mathbf{b} \in \mathbb{R}^{d}$,

$$
(|\mathbf{b}| \mathbf{b}-|\mathbf{a}| \mathbf{a}) \cdot(\mathbf{b}-\mathbf{a}) \geq 0
$$

To prove it, consider the function $J: \mathbb{R}^{d} \mapsto \mathbb{R}$ defined by $J(\mathbf{a})=\frac{2}{3}|\mathbf{a}|^{3} . J$ is convex and differentiable. Thus,

$$
(\nabla J(\mathbf{a})-\nabla J(\mathbf{b})) \cdot(\mathbf{b}-\mathbf{a}) \geq 0, \quad \forall \mathbf{a}, \mathbf{b} \in \mathbb{R}^{d} .
$$

Then, (3.4) follows as $\nabla J(\mathbf{a})=|\mathbf{a}| \mathbf{a} \cdot \mathbf{b}$.

We deduce that

$$
\sum_{i=1}^{2} \frac{1}{\kappa_{i}} \int_{\Omega_{i}} \alpha_{i}\left(k_{i}^{n}\right)\left|\nabla\left(\mathbf{u}_{i}^{n+1}-\mathbf{u}_{i}^{n}\right)\right|^{2} \mathrm{~d} \mathbf{x}+\sum_{i=1}^{2} \frac{1}{\kappa_{i}} \int_{\Omega_{i}}\left(\alpha_{i}\left(k_{i}^{n}\right)-\alpha_{i}\left(k_{i}^{n-1}\right)\right) \nabla \mathbf{u}_{i}^{n} \cdot \nabla\left(\mathbf{u}_{i}^{n+1}-\mathbf{u}_{i}^{n}\right) \mathrm{d} \mathbf{x} \leq 0 .
$$


It comes from Hypothesis 3.1 that $\nabla \mathbf{u}_{i}^{n}$ belongs to $L^{3}\left(\Omega_{i}\right)^{d}$ and that $\left\|\nabla \mathbf{u}_{i}^{n}\right\|_{L^{3}\left(\Omega_{i}\right)^{d}} \leq M$. Furthermore, according to the relation (2.2) and the canonical injection from $H^{1}\left(\Omega_{i}\right)$ to $L^{6}\left(\Omega_{i}\right)$ and Hölder inequality, we obtain

$$
\begin{aligned}
\nu \sum_{i=1}^{2}\left\|\nabla\left(\mathbf{u}_{i}^{n+1}-\mathbf{u}_{i}^{n}\right)\right\|_{L^{2}\left(\Omega_{i}\right)^{d}}^{2} & \leq \delta_{2} \sum_{i=1}^{2} \int_{\Omega_{i}}\left|k_{i}^{n}-k_{i}^{n-1}\right|\left|\nabla \mathbf{u}_{i}^{n}\right|\left|\nabla\left(\mathbf{u}_{i}^{n+1}-\mathbf{u}_{i}^{n}\right)\right| \mathrm{d} \mathbf{x} \\
& \leq \delta_{2} \sum_{i=1}^{2}\left\|k_{i}^{n}-k_{i}^{n-1}\right\|_{L^{6}\left(\Omega_{i}\right)}\left\|\nabla \mathbf{u}_{i}^{n}\right\|_{L^{3}\left(\Omega_{i}\right)^{d}}\left\|\nabla\left(\mathbf{u}_{i}^{n+1}-\mathbf{u}_{i}^{n}\right)\right\|_{L^{2}\left(\Omega_{i}\right)^{d}} \\
& \leq \frac{\delta_{2}^{2} M^{2}}{2 \nu} \sum_{i=1}^{2}\left\|k_{i}^{n}-k_{i}^{n-1}\right\|_{L^{6}\left(\Omega_{i}\right)}^{2}+\frac{\nu}{2} \sum_{i=1}^{2}\left\|\nabla\left(\mathbf{u}_{i}^{n+1}-\mathbf{u}_{i}^{n}\right)\right\|_{L^{2}\left(\Omega_{i}\right)^{d}}^{2} .
\end{aligned}
$$

From this estimate we conclude relation (3.3).

We next prove the contractiveness of the sequence of TKE $\left(k_{i}^{n}\right)_{n}$.

Theorem 3.4. Assume that Hypothesis 3.1 holds and that $\mathbf{f}_{i} \in L^{2}\left(\Omega_{i}\right)^{d}$. Then there exists a positive constant $c$, depending only on $\Omega_{i}$ and on the data $\kappa_{i}$ and $\lambda$, such that for all $n \in \mathbb{N}^{*}$,

$$
\sum_{i=1}^{2}\left\|\nabla\left(k_{i}^{n+1}-k_{i}^{n}\right)\right\|^{2} L^{2}\left(\Omega_{i}\right) \leq c \frac{\left(\delta_{1}^{2}+1\right) \delta_{2}^{2}}{\nu^{3}} M \sum_{i=1}^{2}\left\|\nabla\left(k_{i}^{n}-k_{i}^{n-1}\right)\right\|^{2} L^{2}\left(\Omega_{i}\right) .
$$

Proof. The proof of this theorem is made in several steps.

\section{First step. Choice of the test function}

We first choose a particular test function $\varphi_{i}$ in the equations (2.7)-(2.9). For that purpose, we need to introduce the special space $H_{00}^{\frac{1}{2}}(\Gamma)$ (see [20], Chap. 1, Thm. 11.7 for instance). We also need to introduce the following operator. Let $R_{i}$ be a continuous harmonic lifting operator from $H_{00}^{\frac{1}{2}}(\Gamma)$ to $H^{1}\left(\Omega_{i}\right)$, defined as follows.

For any $\eta$ in $H_{00}^{\frac{1}{2}}(\Gamma), R_{i} \eta$ belongs to $H^{1}\left(\Omega_{i}\right)$, and satisfies

$$
\left\{\begin{aligned}
-\Delta R_{i} \eta & =0 \text { in } \Omega_{i} \\
R_{i} \eta & =\eta \text { on } \Gamma \\
R_{i} \eta & =0 \text { on } \Gamma_{i}
\end{aligned} \quad\right. \text { and }
$$

Moreover, one has

$$
\forall \eta \in H_{00}^{\frac{1}{2}}(\Gamma), \quad\left\|R_{i} \eta\right\|_{H^{1}\left(\Omega_{i}\right)} \leq c_{R}\|\eta\|_{H_{00}^{\frac{1}{2}}(\Gamma)},
$$

where $c_{R}>0$ depends only on $\Omega_{i}$.

According to Hypothesis 3.1, $\forall n \in \mathbb{N}^{*}, k_{i}^{n} \in W^{1,3}\left(\Omega_{i}\right)$, then its trace on $\Gamma$ belongs to $W^{\frac{2}{3}, 3}(\Gamma)$. Thus, by Sobolev's injections, it belongs to $H^{\frac{1}{2}}(\Gamma)$. Furthermore, $k_{i}^{n}=0$ on $\Gamma_{i}$, then $\left.k_{i}^{n}\right|_{\Gamma}$ belongs to $H_{00}^{\frac{1}{2}}(\Gamma)$.

The idea consists in choosing the test function $\varphi_{i}$ equal to $\left(k_{i}^{n+1}-k_{i}^{n}\right)-R_{i}\left(k_{i}^{n+1}-k_{i}^{n}\right)$ in equation (2.9) at steps $n$ and $n+1$. Then, we make the difference between both obtained equations, and sum upon $i=1,2$. We find

$$
\nu \sum_{i=1}^{2}\left\|\nabla\left(k_{i}^{n+1}-k_{i}^{n}\right)\right\|_{0, \Omega_{i}}^{2} \leq \sum_{j=1}^{7} I_{j}
$$


where

$$
\begin{aligned}
& I_{1}=\sum_{i=1}^{2}\left|\int_{\Omega_{i}} \alpha_{i}\left(k_{i}^{n}\right)\left(\left|\nabla \mathbf{u}_{i}^{n+1}\right|^{2}-\left|\nabla \mathbf{u}_{i}^{n}\right|^{2}\right)\left(k_{i}^{n+1}-k_{i}^{n}\right) \mathrm{d} \mathbf{x}\right| \\
& I_{2}=\left.\sum_{i=1}^{2}\left|\int_{\Omega_{i}}\left(\alpha_{i}\left(k_{i}^{n}\right)-\alpha_{i}\left(k_{i}^{n-1}\right)\right)\right| \nabla \mathbf{u}_{i}^{n}\right|^{2}\left(k_{i}^{n+1}-k_{i}^{n}\right) \mathrm{d} \mathbf{x} \mid \\
& I_{3}=\sum_{i=1}^{2}\left|\int_{\Omega_{i}}\left(\gamma_{i}\left(k_{i}^{n}\right)-\gamma_{i}\left(k_{i}^{n-1}\right)\right) \nabla k_{i}^{n} \cdot \nabla\left(k_{i}^{n+1}-k_{i}^{n}\right) \mathrm{d} \mathbf{x}\right| \\
& I_{4}=\sum_{i=1}^{2}\left|\int_{\Omega_{i}}\left(\gamma_{i}\left(k_{i}^{n}\right)-\gamma_{i}\left(k_{i}^{n-1}\right)\right) \nabla k_{i}^{n} \cdot \nabla R_{i}\left(k_{i}^{n+1}-k_{i}^{n}\right) \mathrm{d} \mathbf{x}\right| \\
& I_{5}=\sum_{i=1}^{2}\left|\int_{\Omega_{i}} \alpha_{i}\left(k_{i}^{n}\right)\left(\left|\nabla \mathbf{u}_{i}^{n+1}\right|^{2}-\left|\nabla \mathbf{u}_{i}^{n}\right|^{2}\right) R_{i}\left(k_{i}^{n+1}-k_{i}^{n}\right) \mathrm{d} \mathbf{x}\right| \\
& I_{6}=\left.\sum_{i=1}^{2}\left|\int_{\Omega_{i}}\left(\alpha_{i}\left(k_{i}^{n}\right)-\alpha_{i}\left(k_{i}^{n-1}\right)\right)\right| \nabla \mathbf{u}_{i}^{n}\right|^{2} R_{i}\left(k_{i}^{n+1}-k_{i}^{n}\right) \mathrm{d} \mathbf{x} \mid \\
& I_{7}=\sum_{i=1}^{2}\left|\int_{\Omega_{i}} \gamma_{i}\left(k_{i}^{n}\right) \nabla\left(k_{i}^{n+1}-k_{i}^{n}\right) \cdot \nabla R_{i}\left(k_{i}^{n+1}-k_{i}^{n}\right) \mathrm{d} \mathbf{x}\right|
\end{aligned}
$$

\section{Second step. Estimates of $I_{j}, 1 \leq j \leq 7$}

Estimation of $I_{1}$. We write $\left|\nabla \mathbf{u}_{i}^{n+1}\right|^{2}-\left|\nabla \mathbf{u}_{i}^{n}\right|^{2}=\nabla\left(\mathbf{u}_{i}^{n+1}-\mathbf{u}_{i}^{n}\right) \cdot \nabla\left(\mathbf{u}_{i}^{n+1}+\mathbf{u}_{i}^{n}\right)$, and use Hypothesis 3.1, and relation (2.1). Thanks to the Sobolev embedding of $H^{1}\left(\Omega_{i}\right)$ into $L^{6}\left(\Omega_{i}\right)$ and from Hölder and Poincaré-Friedrichs inequalities, we obtain

$$
\begin{aligned}
& \left|\int_{\Omega_{i}} \alpha_{i}\left(k_{i}^{n}\right)\left(\left|\nabla \mathbf{u}_{i}^{n+1}\right|^{2}-\left|\nabla \mathbf{u}_{i}^{n}\right|^{2}\right)\left(k_{i}^{n+1}-k_{i}^{n}\right) \mathrm{d} \mathbf{x}\right| \\
& \leq \delta_{1}\left\|\nabla\left(\mathbf{u}_{i}^{n+1}-\mathbf{u}_{i}^{n}\right)\right\|_{L^{2}\left(\Omega_{i}\right)^{d}}\left(\left\|\mathbf{u}_{i}^{n+1}\right\|_{W^{1,3}\left(\Omega_{i}\right)^{d}}+\left\|\mathbf{u}_{i}^{n}\right\|_{W^{1,3}\left(\Omega_{i}\right)^{d}}\right)\left\|k_{i}^{n+1}-k_{i}^{n}\right\|_{L^{6}(\Omega)} \\
& \leq M c \delta_{1}\left\|\nabla\left(\mathbf{u}_{i}^{n+1}-\mathbf{u}_{i}^{n}\right)\right\|_{L^{2}\left(\Omega_{i}\right)^{d}}\left\|\nabla\left(k_{i}^{n+1}-k_{i}^{n}\right)\right\|_{L^{2}\left(\Omega_{i}\right)},
\end{aligned}
$$

where $c$ is a positive constant, depending only on domains $\Omega_{i}$. To simplify the calculations, we introduce a positive number $\beta$ which we shall fix later.

According to Young's inequality

$$
\frac{1}{\beta} a^{2}+\beta b^{2} \geq 2 a b, \quad \forall a, b \in \mathbb{R}, \text { and } \forall \beta>0,
$$

we obtain

$\left|\int_{\Omega_{i}} \alpha_{i}\left(k_{i}^{n}\right)\left(\left|\nabla \mathbf{u}_{i}^{n+1}\right|^{2}-\left|\nabla \mathbf{u}_{i}^{n}\right|^{2}\right)\left(k_{i}^{n+1}-k_{i}^{n}\right) \mathrm{d} \mathbf{x}\right| \leq \frac{\nu}{\beta}\left\|\nabla\left(k_{i}^{n+1}-k_{i}^{n}\right)\right\|_{L^{2}\left(\Omega_{i}\right)}^{2}+\frac{\beta M^{2} \delta_{1}^{2} c^{2}}{\nu}\left\|\nabla\left(\mathbf{u}_{i}^{n+1}-\mathbf{u}_{i}^{n}\right)\right\|_{L^{2}\left(\Omega_{i}\right)^{d}}^{2}$. 
Summing upon $i=1,2$, and due to the relation (3.3) from Lemma 3.3, there exists a positive constant $c_{1}$, depending only on $\Omega_{i}, \alpha_{i}$ and $M$, such that

$$
I_{1} \leq \frac{c_{1} \beta \delta_{1}^{2} \delta_{2}^{2} M^{2}}{\nu^{3}} \sum_{i=1}^{2}\left\|\nabla\left(k_{i}^{n}-k_{i}^{n-1}\right)\right\|_{L^{2}\left(\Omega_{i}\right)}^{2}+\frac{\nu}{\beta} \sum_{i=1}^{2}\left\|\nabla\left(k_{i}^{n+1}-k_{i}^{n}\right)\right\|_{L^{2}\left(\Omega_{i}\right)}^{2} .
$$

Estimation of $I_{2}$ and $I_{3}$. Using the same arguments we used for estimation of $I_{1}$, there exists two positive constants, depending only on $\Omega_{i}, \gamma_{i}$ and $M$, such that

$$
I_{2} \leq \frac{c_{2} \beta \delta_{2}^{2} M^{2}}{\nu} \sum_{i=1}^{2}\left\|\nabla\left(k_{i}^{n}-k_{i}^{n-1}\right)\right\|_{L^{2}\left(\Omega_{i}\right)}^{2}+\frac{\nu}{\beta} \sum_{i=1}^{2}\left\|\nabla\left(k_{i}^{n+1}-k_{i}^{n}\right)\right\|_{L^{2}\left(\Omega_{i}\right)}^{2},
$$

and

$$
I_{3} \leq \frac{c_{3} \beta \delta_{2}^{2} M^{2}}{\nu} \sum_{i=1}^{2}\left\|\nabla\left(k_{i}^{n}-k_{i}^{n-1}\right)\right\|_{L^{2}\left(\Omega_{i}\right)}^{2}+\frac{\nu}{\beta} \sum_{i=1}^{2}\left\|\nabla\left(k_{i}^{n+1}-k_{i}^{n}\right)\right\|_{L^{2}\left(\Omega_{i}\right)}^{2} .
$$

Estimation of $I_{4}$. We recall that

$$
I_{4}=\sum_{i=1}^{2}\left|\int_{\Gamma_{i}}\left(\gamma_{i}\left(k_{i}^{n}\right)-\gamma_{i}\left(k_{i}^{n-1}\right)\right) \nabla k_{i}^{n} \cdot \nabla R_{i}\left(k_{i}^{n+1}-k_{i}^{n}\right) \mathrm{d} \mathbf{x}\right| .
$$

Let us apply the Mean Value Theorem to the function $\gamma_{i}$, use relation (2.2) and Hölder inequality. We find

$$
\left|\int_{\Gamma_{i}}\left(\gamma_{i}\left(k_{i}^{n}\right)-\gamma_{i}\left(k_{i}^{n-1}\right)\right) \nabla k_{i}^{n} \cdot \nabla R_{i}\left(k_{i}^{n+1}-k_{i}^{n}\right) \mathrm{d} \mathbf{x}\right| \leq \delta_{2}\left\|k_{i}^{n}-k_{i}^{n-1}\right\|_{L^{6}\left(\Omega_{i}\right)}\left\|\nabla k_{i}^{n}\right\|_{L^{3}\left(\Omega_{i}\right)}\left\|\nabla R_{i}\left(k_{i}^{n+1}-k_{i}^{n}\right)\right\|_{L^{2}\left(\Omega_{i}\right)} .
$$

The continuity of the lifting operator (3.7) and Hypothesis 3.1 imply

$$
\left|\int_{\Gamma_{i}}\left(\gamma_{i}\left(k_{i}^{n}\right)-\gamma_{i}\left(k_{i}^{n-1}\right)\right) \nabla k_{i}^{n} \cdot \nabla R_{i}\left(k_{i}^{n+1}-k_{i}^{n}\right) \mathrm{d} \mathbf{x}\right| \leq c_{R} \delta_{2} M\left\|k_{i}^{n}-k_{i}^{n-1}\right\|_{L^{6}\left(\Omega_{i}\right)}\left\|k_{i}^{n+1}-k_{i}^{n}\right\|_{H_{00}^{\frac{1}{2}}(\Gamma)} .
$$

According to the continuity of the canonical injection from $H^{1}\left(\Omega_{i}\right)$ to $L^{6}\left(\Omega_{i}\right)$, the continuity of the trace operator from $\mathbf{X}_{i}$ to $H_{00}^{\frac{1}{2}}(\Gamma)$, and using Young's inequality (3.9), there exists a positive constant $c_{4}>0$, depending only on $\Omega_{i}, \gamma_{i}$, and $M$, such that

$$
\left|\int_{\Gamma_{i}}\left(\gamma_{i}\left(k_{i}^{n}\right)-\gamma_{i}\left(k_{i}^{n-1}\right)\right) \nabla k_{i}^{n} \cdot \nabla R_{i}\left(k_{i}^{n+1}-k_{i}^{n}\right) \mathrm{d} \mathbf{x}\right| \leq \frac{c_{4} \beta \delta_{2}^{2} M^{2}}{\nu}\left\|\nabla\left(k_{i}^{n}-k_{i}^{n-1}\right)\right\|_{L^{2}\left(\Omega_{i}\right)}^{2}+\frac{\nu}{\beta}\left\|\nabla\left(k_{i}^{n+1}-k_{i}^{n}\right)\right\|_{L^{2}\left(\Omega_{i}\right)}^{2} .
$$

Summing on $i=1,2$, we find

$$
I_{4} \leq \frac{c_{4} \beta \delta_{2}^{2} M^{2}}{\nu} \sum_{i=1}^{2}\left\|\nabla\left(k_{i}^{n}-k_{i}^{n-1}\right)\right\|_{L^{2}\left(\Omega_{i}\right)}^{2}+\frac{\nu}{\beta} \sum_{i=1}^{2}\left\|\nabla\left(k_{i}^{n+1}-k_{i}^{n}\right)\right\|_{L^{2}\left(\Omega_{i}\right)}^{2} .
$$

Estimation of $I_{5}$. We have

$$
I_{5}=\sum_{i=1}^{2}\left|\int_{\Omega_{i}} \alpha_{i}\left(k_{i}^{n}\right)\left(\left|\nabla \mathbf{u}_{i}^{n+1}\right|^{2}-\left|\nabla \mathbf{u}_{i}^{n}\right|^{2}\right) R_{i}\left(k_{i}^{n+1}-k_{i}^{n}\right) \mathrm{d} \mathbf{x}\right| .
$$


Replacing $\left|\nabla \mathbf{u}_{i}^{n+1}\right|^{2}-\left|\nabla \mathbf{u}_{i}^{n}\right|^{2}$ by $\nabla\left(\mathbf{u}_{i}^{n+1}-\mathbf{u}_{i}^{n}\right) \cdot \nabla\left(\mathbf{u}_{i}^{n+1}+\mathbf{u}_{i}^{n}\right)$, and using Hypothesis 3.1, Hölder inequality and formula (3.7), there exists a positive constant $\theta>0$, depending only on $\Omega_{i}$, such that

$$
\left|\int_{\Omega_{i}} \alpha_{i}\left(k_{i}^{n}\right)\left(\left|\nabla \mathbf{u}_{i}^{n+1}\right|^{2}-\left|\nabla \mathbf{u}_{i}^{n}\right|^{2}\right) R_{i}\left(k_{i}^{n+1}-k_{i}^{n}\right) \mathrm{d} \mathbf{x}\right| \leq \theta \delta_{1} M\left\|\nabla\left(\mathbf{u}_{i}^{n+1}-\mathbf{u}_{i}^{n}\right)\right\|_{L^{2}\left(\Omega_{i}\right)^{d}}\left\|\nabla\left(k_{i}^{n+1}-k_{i}^{n}\right)\right\|_{L^{2}\left(\Omega_{i}\right)} .
$$

Then, using relations (3.3) from Lemma 3.3 and (3.9), and summing on $i=1,2$, we obtain the following estimation of $I_{5}$ :

$$
I_{5} \leq \frac{c_{5} \beta \delta_{1}^{2} \delta_{2}^{2} M^{2}}{\nu^{3}} \sum_{i=1}^{2}\left\|\nabla\left(k_{i}^{n}-k_{i}^{n-1}\right)\right\|_{L^{2}\left(\Omega_{i}\right)}^{2}+\frac{\nu}{\beta} \sum_{i=1}^{2}\left\|\nabla\left(k_{i}^{n+1}-k_{i}^{n}\right)\right\|_{L^{2}\left(\Omega_{i}\right)}^{2} .
$$

Estimation of $I_{6}$. Applying the same techniques, we have

$$
\left.\left|\int_{\Omega_{i}}\left(\alpha_{i}\left(k_{i}^{n}\right)-\alpha_{i}\left(k_{i}^{n-1}\right)\right)\right| \nabla \mathbf{u}_{i}^{n}\right|^{2} R_{i}\left(k_{i}^{n+1}-k_{i}^{n}\right) \mathrm{d} \mathbf{x} \mid \leq \delta_{2}\left\|k_{i}^{n}-k_{i}^{n-1}\right\|_{L^{6}\left(\Omega_{i}\right)}\left\|\nabla \mathbf{u}_{i}^{n}\right\|_{L^{3}\left(\Omega_{i}\right)^{d}}^{2}\left\|R_{i}\left(k_{i}^{n+1}-k_{i}^{n}\right)\right\|_{L^{6}\left(\Omega_{i}\right)} .
$$

Using the continuity of the lifting operator $R_{i}$ from $H^{1}$ to $H_{00}^{\frac{1}{2}}(\Gamma)$, the continuity of the canonical injection from $H^{1}\left(\Omega_{i}\right)$ to $L^{6}\left(\Omega_{i}\right)$, and Poincaré-Friedrichs inequality, there exists a positive constant $c_{6}$, depending only on $\Omega_{i}$, such that

$$
\begin{aligned}
\left.\left|\int_{\Omega_{i}}\left(\alpha_{i}\left(k_{i}^{n}\right)-\alpha_{i}\left(k_{i}^{n-1}\right)\right)\right| \nabla \mathbf{u}_{i}^{n}\right|^{2} R_{i}\left(k_{i}^{n+1}-k_{i}^{n}\right) \mathrm{d} \mathbf{x} \mid & \leq c_{6} \delta_{2} M\left\|\nabla\left(k_{i}^{n}-k_{i}^{n-1}\right)\right\|_{L^{2}\left(\Omega_{i}\right)}\left\|\nabla\left(k_{i}^{n+1}-k_{i}^{n}\right)\right\|_{L^{2}\left(\Omega_{i}\right)}, \\
& \leq \frac{c_{6} \beta \delta_{2}^{2} M^{2}}{\nu}\left\|\nabla\left(k_{i}^{n}-k_{i}^{n-1}\right)\right\|_{L^{2}\left(\Omega_{i}\right)}^{2}+\frac{\nu}{\beta}\left\|\nabla\left(k_{i}^{n+1}-k_{i}^{n}\right)\right\|_{L^{2}\left(\Omega_{i}\right)}^{2} .
\end{aligned}
$$

Summing on $i=1,2$, we deduce the following estimation of $I_{6}$,

$$
I_{6} \leq \frac{c_{6} \beta \delta_{2}^{2} M^{2}}{\nu} \sum_{i=1}^{2}\left\|\nabla\left(k_{i}^{n}-k_{i}^{n-1}\right)\right\|_{L^{2}\left(\Omega_{i}\right)}^{2}+\frac{\nu}{\beta} \sum_{i=1}^{2}\left\|\nabla\left(k_{i}^{n+1}-k_{i}^{n}\right)\right\|_{L^{2}\left(\Omega_{i}\right)}^{2} .
$$

Estimation of $I_{7}$. The estimation of $I_{7}$ is more involved. To achieve it, we use a result of continuity of the product of traces on $\Gamma$ due to Grisvard $[13,14]$.

Lemma 3.5. Assume that $\Omega$ is a bounded Lipschitz-continuous open subset of $\mathbb{R}^{d}$. Let $s, s_{1}$ and $s_{2}$ be three non negative reals and $p, p_{1}, p_{2}$ be three real numbers in $[1,+\infty)$ such that $s_{1} \geq s, s_{2} \geq s$ and either

$$
s_{1}+s_{2}-s \geq d\left(\frac{1}{p_{1}}+\frac{1}{p_{2}}-\frac{1}{p}\right) \geq 0, \quad s_{i}-s>d\left(\frac{1}{p_{i}}-\frac{1}{p}\right) \quad i=1,2
$$

or

$$
s_{1}+s_{2}-s>d\left(\frac{1}{p_{1}}+\frac{1}{p_{2}}-\frac{1}{p}\right) \geq 0, \quad s_{i}-s \geq d\left(\frac{1}{p_{i}}-\frac{1}{p}\right) \quad i=1,2 .
$$

Then the mapping $(u, v) \rightarrow u v$ is a continuous bilinear map from $W^{s_{1}, p_{1}}(\Omega) \times W^{s_{2}, p_{2}}(\Omega)$ to $W^{s, p}(\Omega)$.

Using the results of Hebey [15], this lemma also holds for Sobolev spaces defined on compact Riemannian manifolds. This is the case of $\Gamma$.

We remind that

$$
I_{7}=\sum_{i=1}^{2}\left|\int_{\Omega_{i}} \gamma_{i}\left(k_{i}^{n}\right) \nabla\left(k_{i}^{n+1}-k_{i}^{n}\right) \cdot \nabla R_{i}\left(k_{i}^{n+1}-k_{i}^{n}\right) \mathrm{d} \mathbf{x}\right| .
$$


Using relation (2.1), Cauchy-Schwarz inequality, and the continuity of the lifting (3.7), we find

$$
\left|\int_{\Omega_{i}} \gamma_{i}\left(k_{i}^{n}\right) \nabla\left(k_{i}^{n+1}-k_{i}^{n}\right) \cdot \nabla R_{i}\left(k_{i}^{n+1}-k_{i}^{n}\right) \mathrm{d} \mathbf{x}\right| \leq c_{R} \delta_{1}\left\|\nabla\left(k_{i}^{n+1}-k_{i}^{n}\right)\right\|_{L^{2}\left(\Omega_{i}\right)}\left\|k_{i}^{n+1}-k_{i}^{n}\right\|_{\mathbf{H}_{00}^{\frac{1}{2}}(\Gamma)} .
$$

The boundary condition equation (2.7) implies that $\left.\left(k_{i}^{n+1}-k_{i}^{n}\right)\right|_{\Gamma}=\left.\lambda\left(\left|\mathbf{u}_{1}^{n+1}-\mathbf{u}_{2}^{n+1}\right|^{2}-\left|\mathbf{u}_{1}^{n}-\mathbf{u}_{2}^{n}\right|^{2}\right)\right|_{\Gamma}$. Thus

$$
\left\|k_{i}^{n+1}-k_{i}^{n}\right\|_{\mathbf{H}_{00}^{\frac{1}{2}}(\Gamma)}=\lambda\left\|\left[\left(\mathbf{u}_{1}^{n+1}-\mathbf{u}_{1}^{n}\right)-\left(\mathbf{u}_{2}^{n+1}-\mathbf{u}_{2}^{n}\right)\right]\left[\left(\mathbf{u}_{1}^{n+1}+\mathbf{u}_{1}^{n}\right)-\left(\mathbf{u}_{2}^{n+1}+\mathbf{u}_{2}^{n}\right)\right]\right\|_{\mathbf{H}_{00}^{\frac{1}{2}}(\Gamma)} .
$$

Let us apply Lemma 3.5, by taking

$$
\begin{gathered}
s_{1}=s=\frac{1}{2}, \quad s_{2}=1-\frac{1}{3+\varepsilon}, \\
p_{1}=p=2, \quad p_{2}=3+\varepsilon \\
\text { and }\left(\mathbf{u}_{1}^{n+1}-\mathbf{u}_{1}^{n}\right)-\left(\mathbf{u}_{2}^{n+1}-\mathbf{u}_{2}^{n}\right)=\mathbf{u} \in H^{\frac{1}{2}}(\Gamma) \quad\left(=W^{\frac{1}{2}, 2}(\Gamma)=W^{s_{1}, p_{1}}(\Gamma)\right), \\
\left(\mathbf{u}_{1}^{n+1}-\mathbf{u}_{1}^{n}\right)-\left(\mathbf{u}_{2}^{n+1}-\mathbf{u}_{2}^{n}\right)=\mathbf{v} \in W^{1-\frac{1}{3+\varepsilon}, 3+\varepsilon}(\Gamma) \quad\left(=W^{s_{2}, p_{2}}(\Gamma)\right) .
\end{gathered}
$$

We obtain

$$
\begin{aligned}
\left|\int_{\Omega_{i}} \gamma_{i}\left(k_{i}^{n}\right) \nabla\left(k_{i}^{n+1}-k_{i}^{n}\right) \cdot \nabla R_{i}\left(k_{i}^{n+1}-k_{i}^{n}\right) \mathrm{d} \mathbf{x}\right| & \leq c_{R} \lambda \delta_{1}\left\|\nabla\left(k_{i}^{n+1}-k_{i}^{n}\right)\right\| L_{L^{2}\left(\Omega_{i}\right)} \\
& \times\left[\left\|\mathbf{u}_{1}^{n+1}-\mathbf{u}_{1}^{n}\right\|_{\mathbf{H}_{00}^{\frac{1}{2}}(\Gamma)}+\left\|\mathbf{u}_{2}^{n+1}-\mathbf{u}_{2}^{n}\right\|_{\mathbf{H}_{00}^{\frac{1}{2}}(\Gamma)}\right] \\
& \times\left[\left\|\mathbf{u}_{1}^{n+1}+\mathbf{u}_{1}^{n}\right\|_{W^{1-\frac{1}{3+\varepsilon}, 3+\varepsilon}(\Gamma)^{d}}+\left\|\mathbf{u}_{2}^{n+1}+\mathbf{u}_{2}^{n}\right\|_{W^{1-\frac{1}{3+\varepsilon}, 3+\varepsilon}(\Gamma)^{d}}\right] .
\end{aligned}
$$

Using the continuity of the trace operators from $W^{1,3+\varepsilon}\left(\Omega_{i}\right)^{d}$ to $W^{1-\frac{1}{3+\varepsilon}, 3+\varepsilon}(\Gamma)^{d}$ and from $\mathbf{H}^{1}\left(\Omega_{i}\right)$ to $\mathbf{H}_{00}^{\frac{1}{2}}(\Gamma)$, there exists a positive constant $c_{7}^{\prime}$, depending only on domains $\Omega_{i}$ and $\lambda$, such that

$$
\begin{aligned}
\mid \int_{\Omega_{i}} \gamma_{i}\left(k_{i}^{n}\right) & \nabla\left(k_{i}^{n+1}-k_{i}^{n}\right) \cdot \nabla R_{i}\left(k_{i}^{n+1}-k_{i}^{n}\right) \mathrm{d} \mathbf{x} \mid \\
& \leq c_{7}^{\prime} \delta_{1}\left\|\nabla\left(k_{i}^{n+1}-k_{i}^{n}\right)\right\|_{L^{2}\left(\Omega_{i}\right)}\left(\sum_{i=1}^{2}\left\|\mathbf{u}_{i}^{n+1}-\mathbf{u}_{i}^{n}\right\|_{\mathbf{H}^{1}\left(\Omega_{i}\right)^{d}}\right)\left(\sum_{i=1}^{2}\left\|\mathbf{u}_{i}^{n+1}+\mathbf{u}_{i}^{n}\right\|_{W^{1,3+\varepsilon}\left(\Omega_{i}\right)^{d}}\right) \\
& \leq 2 M c_{7}^{\prime} \delta_{1}\left\|\nabla\left(k_{i}^{n+1}-k_{i}^{n}\right)\right\|_{L^{2}\left(\Omega_{i}\right)} \sum_{i=1}^{2}\left\|\mathbf{u}_{i}^{n+1}-\mathbf{u}_{i}^{n}\right\|_{\mathbf{H}^{1}\left(\Omega_{i}\right)^{d}} \quad \text { (by Hypothesis 3.1) } \\
& \leq 2 M c_{7}^{\prime} \delta_{1}\left\|\nabla\left(k_{i}^{n+1}-k_{i}^{n}\right)\right\|_{L^{2}\left(\Omega_{i}\right)} \sum_{i=1}^{2}\left\|\nabla\left(\mathbf{u}_{i}^{n+1}-\mathbf{u}_{i}^{n}\right)\right\|_{L^{2}(\Omega)^{d}} \quad \text { (by Poincaré-Friedrichs inequality). }
\end{aligned}
$$

According to relation (3.3) of Lemma 3.3, relation (3.9) and summing upon $i=1,2$, there exists a positive constant $c_{7}$, depending only on $\Omega_{i}$ and $\lambda$ such that

$$
I_{7} \leq \frac{c_{7} \beta \delta_{1}^{2} M^{2}}{\nu} \sum_{i=1}^{2}\left\|\nabla\left(k_{i}^{n}-k_{i}^{n-1}\right)\right\|_{L^{2}\left(\Omega_{i}\right)}^{2}+\frac{\nu}{\beta} \sum_{i=1}^{2}\left\|\nabla\left(k_{i}^{n+1}-k_{i}^{n}\right)\right\|_{L^{2}\left(\Omega_{i}\right)} .
$$


Finally, using estimations (3.10)-(3.18), relation (3.8), and choosing $\beta=14$ (for instance), there exists a positive constant $c$, depending only on $\Omega_{i}, \kappa_{i}$ and $\lambda$, such that

$$
\sum_{i=1}^{2}\left\|\nabla\left(k_{i}^{n+1}-k_{i}^{n}\right)\right\|_{L^{2}\left(\Omega_{i}\right)}^{2} \leq \frac{c\left(1+\delta_{1}^{2}\right) \delta_{2}^{2} M^{2}}{\nu^{3}} \sum_{i=1}^{2}\left\|\nabla\left(k_{i}^{n}-k_{i}^{n-1}\right)\right\|_{L^{2}\left(\Omega_{i}\right)}^{2} .
$$

This finishes the proof of the Theorem 3.4.

Corollary 3.6 (convergence of the iterative process). Under the hypotheses of Theorem 3.4, there exists a positive constant $c$, depending only on $\Omega_{i}$ and on the data $\kappa_{i}$ and $\lambda$, such that if $K=c \frac{\left(1+\delta_{1}^{2}\right) \delta_{2}^{2}}{\nu^{3}} M^{2}<1$, then the sequences $\left(\mathbf{u}_{i}^{n}\right)_{n}$ and $\left(k_{i}^{n}\right)_{n}$ are contracting, in the sense of relation (3.1), i.e.

$$
\begin{aligned}
& \sum_{i=1}^{2}\left\|\nabla\left(\mathbf{u}_{i}^{n+1}-\mathbf{u}_{i}^{n}\right)\right\|_{L^{2}\left(\Omega_{i}\right)}^{2} \leq K \sum_{i=1}^{2}\left\|\nabla\left(k_{i}^{n}-k_{i}^{n-1}\right)\right\|_{L^{2}\left(\Omega_{i}\right)}^{2}, \quad \text { and } \\
& \sum_{i=1}^{2}\left\|\nabla\left(k_{i}^{n+1}-k_{i}^{n}\right)\right\|_{L^{2}\left(\Omega_{i}\right)}^{2} \leq K \sum_{i=1}^{2}\left\|\nabla\left(k_{i}^{n}-k_{i}^{n-1}\right)\right\|_{L^{2}\left(\Omega_{i}\right)}^{2}
\end{aligned}
$$

Furthermore, from estimate (3.3), as $K<1,\left(\mathbf{u}_{i}^{n}\right)_{n}$ is a Cauchy sequence,

$$
\sum_{i=1}^{2}\left\|\nabla\left(\mathbf{u}_{i}^{m}-\mathbf{u}_{i}^{n}\right)\right\|_{0}^{2} \leq \frac{1-K^{m-n+1}}{1-K} \sum_{i=1}^{2}\left\|\nabla\left(k_{i}^{n}-k_{i}^{n-1}\right)\right\|_{0}^{2}, \quad \forall n \leq m \in \mathbb{N}
$$

Then, since $\mathbf{X}_{i}$ and $H^{1}\left(\Omega_{i}\right)$ are Banach spaces, the sequences $\left(\mathbf{u}_{i}^{n}\right)_{n}$ and $\left(k_{i}^{n}\right)_{n}$ have unique strong limits in $\mathbf{X}_{i}$ and $H^{1}\left(\Omega_{i}\right)$, $\mathbf{u}_{i}$ and $k_{i}$.

We next prove that the sequence $\left(p_{i}^{n}\right)_{n}$ is a Cauchy sequence. For that purpose, we use the following inf-sup condition proved in [3], Lemma 3.1.

Corollary 3.7 (inf-sup condition). Assume that $\Omega_{i}$ is bounded Lipschitz-continuous open subset of $\mathbb{R}^{d}$. Then, there exists a positive constant $\beta_{i}>0$, depending only on the domain $\Omega_{i}$, such that

$$
\forall q_{i} \in L^{2}\left(\Omega_{i}\right), \quad \sup _{\mathbf{v}_{i} \in \mathbf{X}_{i}} \frac{b_{i}\left(\mathbf{v}_{i}, q_{i}\right)}{\left\|\mathbf{v}_{i}\right\|_{\mathbf{H}^{1}\left(\Omega_{i}\right)}} \geq \beta_{i}\left\|q_{i}\right\|_{L^{2}\left(\Omega_{i}\right)} .
$$

Theorem 3.8 (convergence of the pressure). Assume that Hypothesis 3.1 holds, that $\mathbf{f}_{i} \in L^{2}\left(\Omega_{i}\right)^{d}$ and that $K<1$. Then, $\left(p_{i}^{n}\right)_{n}$ is a Cauchy sequence. More specifically, there exists positive constants $c^{\prime}$, depending only on $\Omega_{i}, \alpha_{i}$, and $M$, and $c^{\prime \prime}$ depending only on $\Omega_{i}$, such that for any two non negative integers $m \geq n$,

$$
\begin{aligned}
\sum_{i=1}^{2}\left\|p_{i}^{m+1}-p_{i}^{n+1}\right\|_{0}^{2} \leq & \frac{c^{\prime}}{\beta^{2}(1-K)} \sum_{i=1}^{2}\left\|\nabla\left(k_{i}^{n+1}-k_{i}^{n}\right)\right\|_{0}^{2} \\
& +\frac{c^{\prime \prime}}{\beta^{2}}\left\|\left|\mathbf{u}_{1}^{m+1}-\mathbf{u}_{2}^{m+1}\right|\left(\mathbf{u}_{1}^{m+1}-\mathbf{u}_{2}^{m+1}\right)-\left|\mathbf{u}_{1}^{n+1}-\mathbf{u}_{2}^{n+1}\right|\left(\mathbf{u}_{1}^{n+1}-\mathbf{u}_{2}^{n+1}\right)\right\|_{L^{\frac{3}{2}}(\Gamma)^{d}}^{2}
\end{aligned}
$$

where $\beta=\min \left\{\beta_{1}, \beta_{2}\right\}$. 
Proof. For all $\mathbf{v}_{i} \in \mathbf{X}_{i}$, we make the difference of equation (2.5) at steps $m$ and $n$. This yields for $1 \leq i \neq j \leq 2$,

$$
\begin{aligned}
\frac{b_{i}\left(\mathbf{v}_{i}, p_{i}^{m+1}-p_{i}^{n+1}\right)}{\left\|\mathbf{v}_{i}\right\|_{\mathbf{H}^{1}\left(\Omega_{i}\right)^{d}}}= & -\kappa_{i} \int_{\Gamma}\left[\left|\mathbf{u}_{i}^{m+1}-\mathbf{u}_{j}^{m+1}\right|\left(\mathbf{u}_{i}^{m+1}-\mathbf{u}_{j}^{m+1}\right)-\left|\mathbf{u}_{i}^{n+1}-\mathbf{u}_{j}^{n+1}\right|\left(\mathbf{u}_{i}^{n+1}-\mathbf{u}_{j}^{n+1}\right)\right] \cdot \frac{\mathbf{v}_{i}}{\left\|\mathbf{v}_{i}\right\|_{\mathbf{H}^{1}\left(\Omega_{i}\right)^{d}}} \mathrm{~d} \tau \\
& -\int_{\Omega_{i}} \alpha_{i}\left(k_{i}^{m}\right) \nabla\left(\mathbf{u}_{i}^{m+1}-\mathbf{u}_{i}^{n+1}\right): \frac{\nabla \mathbf{v}_{i}}{\left\|\mathbf{v}_{i}\right\|_{\mathbf{H}^{1}\left(\Omega_{i}\right)^{d}}} \mathrm{~d} \mathbf{x} \\
& -\int_{\Omega_{i}}\left(\alpha_{i}\left(k_{i}^{m}\right)-\alpha_{i}\left(k_{i}^{n}\right)\right) \nabla \mathbf{u}_{i}^{n}: \frac{\nabla \mathbf{v}_{i}}{\left\|\mathbf{v}_{i}\right\|_{\mathbf{H}^{1}\left(\Omega_{i}\right)^{d}}} \mathrm{~d} \mathbf{x} .
\end{aligned}
$$

We know that the sequence $\left(\mathbf{u}_{i}^{n}\right)_{n}$ belongs to $\mathbf{H}^{1}\left(\Omega_{i}\right)$, then its trace on $\gamma$ belongs to $\mathbf{H}^{\frac{1}{2}}(\Gamma)$, thus in $L^{3}(\Gamma)^{d}$ by injection, i.e.

$$
|| \mathbf{u}_{i}^{m+1}-\mathbf{u}_{j}^{m+1}\left|\left(\mathbf{u}_{i}^{m+1}-\mathbf{u}_{j}^{m+1}\right)-\right| \mathbf{u}_{i}^{n+1}-\mathbf{u}_{j}^{n+1}\left|\left(\mathbf{u}_{i}^{n+1}-\mathbf{u}_{j}^{n+1}\right)\right| \in L^{\frac{3}{2}}(\Gamma)^{d}, \quad \forall i \neq j \text { and } n \leq m .
$$

Using the Mean Value Theorem, relations (2.1)-(2.2) and Hölder inequality, we find

$$
\begin{aligned}
\frac{b_{i}\left(\mathbf{v}_{i}, p_{i}^{m+1}-p_{i}^{n+1}\right)}{\left\|\mathbf{v}_{i}\right\|_{\mathbf{H}^{1}\left(\Omega_{i}\right)}} \leq & \left\|\left|\mathbf{u}_{i}^{m+1}-\mathbf{u}_{j}^{m+1}\right|\left(\mathbf{u}_{i}^{m+1}-\mathbf{u}_{j}^{m+1}\right)-\left|\mathbf{u}_{i}^{n+1}-\mathbf{u}_{j}^{n+1}\right|\left(\mathbf{u}_{i}^{n+1}-\mathbf{u}_{j}^{n+1}\right)\right\|_{L^{\frac{3}{2}}(\Gamma)^{d}} \frac{\left\|\mathbf{v}_{i}\right\|_{\left.L^{3}(\Gamma)\right)^{d}}}{\left\|\mathbf{v}_{i}\right\|_{\mathbf{H}^{1}\left(\Omega_{i}\right)}} \\
& +\delta_{1}\left\|\nabla\left(\mathbf{u}_{i}^{m+1}-\mathbf{u}_{i}^{n+1}\right)\right\|_{L^{2}\left(\Omega_{i}\right)^{d}} \frac{\left\|\nabla \mathbf{v}_{i}\right\|_{L^{2}\left(\Omega_{i}\right)^{d}}}{\left\|\mathbf{v}_{i}\right\|_{\mathbf{H}^{1}\left(\Omega_{i}\right)}} \\
& +\delta_{2}\left\|k_{i}^{m}-k_{i}^{n}\right\|_{L^{6}\left(\Omega_{i}\right)}\left\|\nabla \mathbf{u}_{i}^{n}\right\|_{L^{3}\left(\Omega_{i}\right)^{d}} \frac{\left\|\nabla \mathbf{v}_{i}\right\|_{L^{2}\left(\Omega_{i}\right)^{d}}}{\left\|\mathbf{v}_{i}\right\|_{\mathbf{H}^{1}\left(\Omega_{i}\right)}}
\end{aligned}
$$

Applying the continuity of the trace operator from $\mathbf{H}^{1}\left(\Omega_{i}\right)$ to $\mathbf{H}^{\frac{1}{2}}(\Gamma)$, and the continuity of the canonical injection from $\mathbf{H}^{\frac{1}{2}}(\Gamma)$ to $L^{3}(\Gamma)^{d}$, there exists a positive constant $c$, depending only on $\Omega_{i}, \alpha_{i}$ and $M$, such that for all $\mathbf{v}_{i} \in \mathbf{X}_{i}$

$$
\begin{aligned}
\frac{b_{i}\left(\mathbf{v}_{i}, p_{i}^{m+1}-p_{i}^{n+1}\right)}{\left\|\mathbf{v}_{i}\right\|_{\mathbf{H}^{1}\left(\Omega_{i}\right)}} \leq & c\left(\left\|\left|\mathbf{u}_{i}^{m+1}-\mathbf{u}_{j}^{m+1}\right|\left(\mathbf{u}_{i}^{m+1}-\mathbf{u}_{j}^{m+1}\right)-\left|\mathbf{u}_{i}^{n+1}-\mathbf{u}_{j}^{n+1}\right|\left(\mathbf{u}_{i}^{n+1}-\mathbf{u}_{j}^{n+1}\right)\right\|_{L^{\frac{3}{2}}(\Gamma)^{d}}\right. \\
& \left.+\left\|\nabla\left(\mathbf{u}_{i}^{m+1}-\mathbf{u}_{i}^{n+1}\right)\right\|_{L^{2}\left(\Omega_{i}\right)^{d}}+\left\|\nabla\left(k_{i}^{m}-k_{i}^{n}\right)\right\|_{L^{2}\left(\Omega_{i}\right)}\left\|\nabla \mathbf{u}_{i}^{n}\right\|_{L^{3}\left(\Omega_{i}\right)^{d}}\right) .
\end{aligned}
$$

Using the inf-sup condition (Cor. 3.7), and summing on $i=1,2$, we obtain

$$
\begin{aligned}
\beta \sum_{i=1}^{2}\left\|p_{i}^{m+1}-p_{i}^{n+1}\right\|_{L^{2}\left(\Omega_{i}\right)} \leq & c\left[\left\|\left|\mathbf{u}_{1}^{m+1}-\mathbf{u}_{2}^{m+1}\right|\left(\mathbf{u}_{1}^{m+1}-\mathbf{u}_{2}^{m+1}\right)-\left|\mathbf{u}_{1}^{n+1}-\mathbf{u}_{2}^{n+1}\right|\left(\mathbf{u}_{1}^{n+1}-\mathbf{u}_{2}^{n+1}\right)\right\|_{L^{\frac{3}{2}}(\Gamma)^{d}}\right. \\
& \left.+\sqrt{2}\left(\sum_{i=1}^{2}\left\|\nabla\left(\mathbf{u}_{i}^{m+1}-\mathbf{u}_{i}^{n+1}\right)\right\|_{L^{2}\left(\Omega_{i}\right)^{d}}^{2}\right)^{\frac{1}{2}}+\sqrt{2}\left(\sum_{i=1}^{2}\left\|\nabla\left(k_{i}^{m}-k_{i}^{n}\right)\right\|_{L^{2}\left(\Omega_{i}\right)}^{2}\right)^{\frac{1}{2}}\right] .
\end{aligned}
$$

According to relation (3.19)

$$
\begin{aligned}
\sum_{i=1}^{2}\left\|p_{i}^{m+1}-p_{i}^{n+1}\right\|_{L^{2}\left(\Omega_{i}\right)}^{2} & \leq 4 \sqrt{2} \frac{c^{2}}{\beta^{2}(1-K)} \sum_{i=1}^{2}\left\|\nabla\left(k_{i}^{n+1}-k_{i}^{n}\right)\right\|_{L^{2}\left(\Omega_{i}\right)}^{2} \\
& +\frac{2 c^{2}}{\beta^{2}}\left\|\left|\mathbf{u}_{1}^{m+1}-\mathbf{u}_{2}^{m+1}\right|\left(\mathbf{u}_{1}^{m+1}-\mathbf{u}_{2}^{m+1}\right)-\left|\mathbf{u}_{1}^{n+1}-\mathbf{u}_{2}^{n+1}\right|\left(\mathbf{u}_{1}^{n+1}-\mathbf{u}_{2}^{n+1}\right)\right\|_{L^{\frac{3}{2}}(\Gamma)^{d}}^{2}
\end{aligned}
$$


We have proved that the sequence $\left(\mathbf{u}_{i}^{n}\right)_{n}$ converges in $\mathbf{H}^{1}\left(\Omega_{i}\right)$ strong. Then, using the continuity of the trace operator from $\mathbf{H}^{1}\left(\Omega_{i}\right)$ to $\mathbf{H}^{\frac{1}{2}}(\Gamma)$, and that of the canonical injection from $\mathbf{H}^{\frac{1}{2}}(\Gamma)$ into $L^{3}(\Gamma)^{d}$, we deduce that the sequence

is a Cauchy sequence in $L^{\frac{3}{2}}(\Gamma)^{d}$. Thus

$$
\left(\left[\left(\mathbf{u}_{1}^{n}-\mathbf{u}_{2}^{n}\right)\left|\mathbf{u}_{1}^{n}-\mathbf{u}_{2}^{n}\right|\right]_{\left.\right|_{\Gamma}}\right)_{n}
$$

$$
\|\left|\mathbf{u}_{1}^{m+1}-\mathbf{u}_{2}^{m+1}\right|\left(\mathbf{u}_{1}^{m+1}-\mathbf{u}_{2}^{m+1}\right)-\left.\left|\mathbf{u}_{1}^{n+1}-\mathbf{u}_{2}^{n+1}\right|\left(\mathbf{u}_{1}^{n+1}-\mathbf{u}_{2}^{n+1}\right)\right|_{L^{\frac{3}{2}}(\Gamma)^{d}} ^{2} \longrightarrow 0 .
$$

We conclude that $\left(p_{i}^{n}\right)_{n}$ is a Cauchy sequence in $L^{2}\left(\Omega_{i}\right), i=1,2$.

We shall denote by $p_{i}$ the limit of the sequence $\left(p_{i}^{n}\right)_{n}$.

\section{IDENTIFICATION OF THE Limit}

In this section, we show that the limit $\left(\mathbf{u}_{i}, p_{i}, k_{i}\right)$ of the sequence $\left(\mathbf{u}_{i}^{n}, p_{i}^{n}, k_{i}^{n}\right)_{n}$ is a solution of the variational formulation (2.3)-(2.4).

Theorem 4.1 (identification of the limit). Under the same assumptions of Theorem 3.6, the limit $\left(\mathbf{u}_{i}, p_{i}, k_{i}\right)$ of the sequence $\left(\mathbf{u}_{i}^{n}, p_{i}^{n}, k_{i}^{n}\right)_{n}$ is a solution of the variational formulation (2.3)-(2.4).

Proof. The proof is made of three steps:

- Stokes equation $(2.3)$ is verified by $\left(\mathbf{u}_{i}, p_{i}, k_{i}\right)$;

- The TKE equation (2.4) is verified by $\left(\mathbf{u}_{i}, p_{i}, k_{i}\right)$; and

- The boundary conditions are verified, i.e. $k_{i}=\lambda\left|\mathbf{u}_{1}-\mathbf{u}_{2}\right|^{2}$ on $\Gamma$ and $k_{i}$ vanishes on $\Gamma_{i}$.

\section{First step. Stokes equation}

Let $\mathbf{v}_{i} \in \mathbf{X}_{i}$, and let us show that

$$
a_{i}\left(k_{i}, \mathbf{u}_{i}, \mathbf{v}_{i}\right)+b_{i}\left(\mathbf{v}_{i}, p_{i}\right)+\kappa_{i} \int_{\Gamma}\left|\mathbf{u}_{i}-\mathbf{u}_{j}\right|\left(\mathbf{u}_{i}-\mathbf{u}_{j}\right) \mathbf{v}_{i} \mathrm{~d} \tau=\int_{\Omega_{i}} \mathbf{f}_{i} \mathbf{v}_{i} \mathrm{~d} \mathbf{x}, \quad \forall 1 \leq i \neq j \leq 2 .
$$

We first focus on the bilinear form $a_{i}(\cdot ; \cdot, \cdot) . k_{i}$ is the limit of the sequence $\left(k_{i}^{n}\right)_{n}$ in $H^{1}\left(\Omega_{i}\right)$. Then, there exists a subsequence of $\left(k_{i}^{n}\right)_{n}$ that converges a.e. in $\Omega_{i}$ to $k_{i}$. As the global sequence is contracting, all the subsequences are convergent and converge to $k_{i}$. Thus, as the function $\alpha_{i}$ is continuous and bounded, $\lim _{n \rightarrow \infty} \alpha_{i}\left(k_{i}^{n}\right)=\alpha_{i}\left(k_{i}\right)$, a.e. in $\Omega_{i}$. Moreover, the sequence $\left(\mathbf{u}_{i}^{n}\right)_{n}$ converges strongly to the unique limit $\mathbf{u}_{i}$ in $\mathbf{H}^{1}\left(\Omega_{i}\right)$, then $\lim _{n \rightarrow \infty} \nabla \mathbf{u}_{i}^{n}=\nabla \mathbf{u}_{i}$ strongly in $L^{2}\left(\omega_{i}\right)^{d}$. Hence by the inverse Lebesgue theorem (see for instance [7], Thm. IV.9), there exists a further subsequence, still denoted by $\left(\nabla \mathbf{u}_{i}^{n}\right)_{n}$, which tends to $\nabla \mathbf{u}_{i}$ a.e. on $\Omega_{i}$, and $\forall n \in \mathbb{N},\left|\nabla \mathbf{u}_{i}\right| \leq g_{i}$, where the function $g_{i}$ belongs to $L^{2}\left(\Omega_{i}\right)$. Thus $\forall \mathbf{v}_{i} \in \mathbf{X}_{i}$,

$$
\begin{gathered}
\lim _{n \rightarrow \infty} \alpha_{i}\left(k_{i}^{n}\right) \nabla \mathbf{u}_{i}^{n}: \nabla \mathbf{v}_{i}=\alpha_{i}\left(k_{i}\right) \nabla \mathbf{u}_{i}: \nabla \mathbf{v}_{i}, \text { a.e. on } \Omega_{i}, \text { and } \\
\left|\alpha_{i}\left(k_{i}^{n}\right) \nabla \mathbf{u}_{i}^{n}: \nabla \mathbf{v}_{i}\right| \leq \delta_{1}\left|g_{i} \| \nabla \mathbf{v}_{i}\right| \in L^{1}\left(\Omega_{i}\right) .
\end{gathered}
$$

Again, using the uniqueness of the limit of all subsequences of $\left(\mathbf{u}_{i}^{n}\right)_{n}$, we obtain $\lim _{n \rightarrow \infty} a_{i}\left(k_{i}^{n} ; \mathbf{u}_{i}^{n+1}, \mathbf{v}_{i}\right)=$ $a_{i}\left(k_{i} ; \mathbf{u}_{i}, \mathbf{v}_{i}\right)$.

Due to the strong convergence of the sequence $\left(p_{i}^{n}\right)$ to $p_{i}$ in $L^{2}\left(\Omega_{i}\right)$, we have $\lim _{n \rightarrow \infty} b_{i}\left(\mathbf{v}_{i}, p_{i}^{n+1}\right)=b_{i}\left(\mathbf{v}_{i}, p_{i}\right)$, for each vector $\mathbf{v}_{i}$ that belongs to $\mathbf{X}_{i}$. 
Concerning the boundary term, since the trace of $\mathbf{u}_{i}^{n}$ on $\Gamma$ belongs to $\mathbf{H}^{\frac{1}{2}}(\Gamma)$ and using the compactness of the canonical injection of $\mathbf{H}^{\frac{1}{2}}(\Gamma)$ to $L^{3}(\Gamma)^{d}$, we have $\lim _{n \rightarrow \infty} \mathbf{u}_{i}^{n}=\mathbf{u}_{i}$ in $L^{3}(\Gamma)^{d}$, thus for $1 \leq i \neq j \leq 2$

$$
\lim _{n \rightarrow \infty}\left|\mathbf{u}_{i}^{n+1}-\mathbf{u}_{j}^{n+1}\right|\left(\mathbf{u}_{i}^{n+1}-\mathbf{u}_{j}^{n+1}\right)=\left|\mathbf{u}_{i}-\mathbf{u}_{j}\right|\left(\mathbf{u}_{i}-\mathbf{u}_{j}\right), \quad \text { strongly in } L^{\frac{3}{2}}(\Gamma)^{d} .
$$

Furthermore, the trace of the vector $\mathbf{v}_{i}$ belongs to $L^{3}(\Gamma)^{d}$, then

$$
\lim _{n \rightarrow \infty} \kappa_{i} \int_{\Gamma}\left|\mathbf{u}_{i}^{n+1}-\mathbf{u}_{j}^{n+1}\right|\left(\mathbf{u}_{i}^{n+1}-\mathbf{u}_{j}^{n+1}\right) \mathbf{v}_{i} \mathrm{~d} \tau=\kappa_{i} \int_{\Gamma}\left|\mathbf{u}_{i}-\mathbf{u}_{j}\right|\left(\mathbf{u}_{i}-\mathbf{u}_{j}\right) \mathbf{v}_{i} \mathrm{~d} \tau
$$

We proved that the limit $\left(\mathbf{u}_{i}, p_{i}, k_{i}\right)$ of the sequence $\left(\mathbf{u}_{i}^{n}, p_{i}^{n}, k_{i}^{n}\right)_{n}$ verifies the first equation of the Stokes problem (2.3). Concerning the second one, due to the fact that $b_{i}\left(\mathbf{u}_{i}^{n+1}, q_{i}\right)=0, \forall q_{i} \in L^{2}\left(\Omega_{i}\right)$, and to the strong convergence in $\mathbf{H}^{1}\left(\Omega_{i}\right)$ of the sequence $\left(\mathbf{u}_{i}^{n}\right)_{n}$ to $\mathbf{u}_{i}$, we deduce that $b_{i}\left(\mathbf{u}_{i}, q_{i}\right)=0, \forall q_{i} \in L^{2}\left(\Omega_{i}\right)$.

\section{Second step. Equation for the TKE}

We want to show that $\forall \varphi_{i} \in W_{0}^{1, r}\left(\Omega_{i}\right), \int_{\Omega_{i}} \gamma_{i}\left(k_{i}\right) \nabla k_{i} \cdot \nabla \varphi_{i} \mathrm{~d} \mathbf{x}=\int_{\Omega_{i}} \alpha_{i}\left(k_{i}\right)\left|\nabla \mathbf{u}_{i}\right|^{2} \varphi_{i} \mathrm{~d} \mathbf{x}$. To achieve this result, we only need to show that

$$
\begin{aligned}
& \lim _{n \rightarrow \infty} \int_{\Omega_{i}} \gamma_{i}\left(k_{i}^{n}\right) \nabla k_{i}^{n+1} \cdot \nabla \varphi_{i} \mathrm{~d} \mathbf{x}=\int_{\Omega_{i}} \gamma_{i}\left(k_{i}\right) \nabla k_{i} \cdot \nabla \varphi_{i} \mathrm{~d} \mathbf{x}, \quad \text { and } \\
& \lim _{n \rightarrow \infty} \int_{\Omega_{i}} \alpha_{i}\left(k_{i}^{n}\right)\left|\nabla \mathbf{u}_{i}^{n+1}\right|^{2} \varphi_{i} \mathrm{~d} \mathbf{x}=\int_{\Omega_{i}} \alpha_{i}\left(k_{i}\right)\left|\nabla \mathbf{u}_{i}\right|^{2} \varphi_{i} \mathrm{~d} \mathbf{x} .
\end{aligned}
$$

The idea consists in choosing test functions $\varphi_{i}$ that belong to $\mathcal{D}\left(\Omega_{i}\right)$ at first, then by density we will consider all test functions in $W_{0}^{1, r}\left(\Omega_{i}\right)$.

Let us write $\gamma_{i}\left(k_{i}^{n}\right) \nabla k_{i}^{n+1}-\gamma_{i}\left(k_{i}\right) \nabla k_{i}$ using (4.1). For all $\varphi_{i} \in \mathcal{D}\left(\Omega_{i}\right)$ :

$$
\int_{\Omega_{i}}\left(\gamma_{i}\left(k_{i}^{n}\right) \nabla k_{i}^{n+1}-\gamma_{i}\left(k_{i}\right) \nabla k_{i}\right) \cdot \nabla \varphi_{i} \mathrm{~d} \mathbf{x}=\int_{\Omega_{i}} \gamma_{i}\left(k_{i}^{n}\right) \nabla\left(k_{i}^{n+1}-k_{i}\right) \cdot \nabla \varphi_{i} \mathrm{~d} \mathbf{x}+\int_{\Omega_{i}}\left(\gamma_{i}\left(k_{i}^{n}\right)-\gamma_{i}\left(k_{i}\right)\right) \nabla k_{i} \cdot \nabla \varphi_{i} \mathrm{~d} \mathbf{x} .
$$

Using the Cauchy-Schwarz inequality, and the strong convergence of the sequence $\left(k_{i}^{n}\right)_{n}$ to $k_{i}$ in $H^{1}\left(\Omega_{i}\right)$, we obtain

$$
\left|\int_{\Omega_{i}} \gamma_{i}\left(k_{i}^{n}\right) \nabla\left(k_{i}^{n+1}-k_{i}\right) \cdot \nabla \varphi_{i} \mathrm{~d} \mathbf{x}\right| \leq \delta_{1}\left\|\nabla\left(k_{i}^{n+1}-k_{i}\right)\right\|_{L^{2}\left(\Omega_{i}\right)}\left\|\nabla \varphi_{i}\right\|_{L^{2}\left(\Omega_{i}\right)} \underset{n \rightarrow \infty}{\longrightarrow} 0 .
$$

On the other hand, we apply the Mean Value Theorem to the function $\gamma_{i}$, and using Hölder inequality, we find

$$
\left|\int_{\Omega_{i}}\left(\gamma_{i}\left(k_{i}^{n}\right)-\gamma_{i}\left(k_{i}\right)\right) \nabla k_{i} \cdot \nabla \varphi_{i} \mathrm{~d} \mathbf{x}\right| \leq \delta_{2}\left\|k_{i}^{n}-k_{i}\right\|_{L^{2}\left(\Omega_{i}\right)}\left\|\nabla k_{i}\right\|_{L^{2}\left(\Omega_{i}\right)}\left\|\nabla \varphi_{i}\right\|_{\infty} \underset{n \rightarrow \infty}{\longrightarrow} 0 .
$$

We deduce that for all $\varphi_{i} \in \mathcal{D}\left(\Omega_{i}\right), \lim _{n \rightarrow \infty} \int_{\Omega_{i}} \gamma_{i}\left(k_{i}^{n}\right) \nabla k_{i}^{n+1} \cdot \nabla \varphi_{i} \mathrm{~d} \mathbf{x}=\int_{\Omega_{i}} \gamma_{i}\left(k_{i}\right) \nabla k_{i} \cdot \nabla \varphi_{i} \mathrm{~d} \mathbf{x}$. Let $\eta$ be a strictly positive real, such that for all integer $n \geq n_{0}$, we have

$$
\left|\int_{\Omega_{i}} \gamma_{i}\left(k_{i}^{n}\right) \nabla k_{i}^{n+1} \cdot \nabla \varphi_{i} \mathrm{~d} \mathbf{x}-\int_{\Omega_{i}} \gamma_{i}\left(k_{i}\right) \nabla k_{i} \cdot \nabla \varphi_{i} \mathrm{~d} \mathbf{x}\right| \leq \frac{\eta}{3}, \quad \forall \varphi_{i} \in \mathcal{D}(\Omega) .
$$

Let now $\varphi_{i}$ belong to $W_{0}^{1, r}\left(\Omega_{i}\right)$. Since $\mathcal{D}\left(\Omega_{i}\right)$ is dense in $W_{0}^{1, r}\left(\Omega_{i}\right)$, there exists a sequence $\left(\varphi_{i}^{m}\right)_{m} \geq 0$ that belongs to $\mathcal{D}\left(\Omega_{i}\right)$, such that for $m \geq m_{0}$, we have $\left\|\varphi_{i}^{m}-\varphi_{i}\right\|_{W_{0}^{1, r}\left(\Omega_{i}\right)} \leq \frac{\eta}{3}$. Thanks to the Sobolev continuous 
embedding from $W_{0}^{1, r}\left(\Omega_{i}\right)$ to $H_{0}^{1}(\Omega)$, we can write for $m \geq m_{0}$

$$
\left\|\varphi_{i}^{m}-\varphi_{i}\right\|_{H_{0}^{1}\left(\Omega_{i}\right)} \leq \frac{\eta}{3}
$$

Thanks to the triangular inequality, we obtain

$$
\begin{aligned}
\left|\int_{\Omega_{i}} \gamma_{i}\left(k_{i}^{n}\right) \nabla k_{i}^{n+1} \cdot \nabla \varphi_{i} \mathrm{~d} \mathbf{x}-\int_{\Omega_{i}} \gamma_{i}\left(k_{i}\right) \nabla k_{i} \cdot \nabla \varphi_{i} \mathrm{~d} \mathbf{x}\right| \leq & \left|\int_{\Omega_{i}} \gamma_{i}\left(k_{i}^{n}\right) \nabla k_{i}^{n+1} \cdot \nabla \varphi_{i} \mathrm{~d} \mathbf{x}-\int_{\Omega_{i}} \gamma_{i}\left(k_{i}^{n}\right) \nabla k_{i}^{n+1} \cdot \nabla \varphi_{i}^{m} \mathrm{~d} \mathbf{x}\right| \\
& +\left|\int_{\Omega_{i}} \gamma_{i}\left(k_{i}^{n}\right) \nabla k_{i}^{n+1} \cdot \nabla \varphi_{i}^{m} \mathrm{~d} \mathbf{x}-\int_{\Omega_{i}} \gamma_{i}\left(k_{i}\right) \nabla\left(k_{i}\right) \cdot \nabla \varphi_{i}^{m} \mathrm{~d} \mathbf{x}\right| \\
& +\left|\int_{\Omega_{i}} \gamma_{i}\left(k_{i}\right) \nabla\left(k_{i}\right) \cdot \nabla \varphi_{i}^{m} \mathrm{~d} \mathbf{x}-\int_{\Omega_{i}} \gamma_{i}\left(k_{i}\right) \nabla\left(k_{i}\right) \cdot \nabla \varphi_{i} \mathrm{~d} \mathbf{x}\right| .
\end{aligned}
$$

Hölder inequality implies

$$
\begin{aligned}
\mid \int_{\Omega_{i}} \gamma_{i}\left(k_{i}^{n}\right) \nabla k_{i}^{n+1} \cdot & \nabla \varphi_{i} \mathrm{~d} \mathbf{x}-\int_{\Omega_{i}} \gamma_{i}\left(k_{i}\right) \nabla k_{i} \cdot \nabla \varphi_{i} \mathrm{~d} \mathbf{x} \mid \leq \delta_{1}\left\|\nabla k_{i}^{n+1}\right\|_{L^{2}\left(\Omega_{i}\right)}\left\|\nabla\left(\varphi_{i}^{m}-\varphi_{i}\right)\right\|_{L^{2}\left(\Omega_{i}\right)} \\
& +\left|\int_{\Omega_{i}} \gamma_{i}\left(k_{i}^{n}\right) \nabla k_{i}^{n+1} \cdot \nabla \varphi_{i}^{m} \mathrm{~d} \mathbf{x}-\int_{\Omega_{i}} \gamma_{i}\left(k_{i}\right) \nabla\left(k_{i}\right) \cdot \nabla \varphi_{i}^{m} \mathrm{~d} \mathbf{x}\right|+\delta_{1}\left\|\nabla k_{i}\right\|_{0}\left\|\nabla\left(\varphi_{i}^{m}-\varphi_{i}\right)\right\|_{L^{2}\left(\Omega_{i}\right)} .
\end{aligned}
$$

Using relations (4.2)-(4.3), and the strong convergence of the sequence $\left(k_{i}^{n}\right)_{n}$ in $H^{1}\left(\Omega_{i}\right)$, we deduce that for any function $\varphi_{i} \in W_{0}^{1, r}\left(\Omega_{i}\right)$, we have

$$
\lim _{n \rightarrow \infty} \int_{\Omega_{i}} \gamma_{i}\left(k_{i}^{n}\right) \nabla\left(k_{i}^{n+1}\right) \cdot \nabla \varphi_{i} \mathrm{~d} \mathbf{x}=\int_{\Omega_{i}} \gamma_{i}\left(k_{i}\right) \nabla\left(k_{i}\right) \cdot \nabla \varphi_{i} \mathrm{~d} \mathbf{x} .
$$

To show the second equation of the relation (4.1), we write

$$
\begin{aligned}
& \left.\left|\int_{\Omega_{i}} \alpha_{i}\left(k_{i}^{n}\right)\right| \nabla \mathbf{u}_{i}^{n+1}\right|^{2}-\alpha_{i}\left(k_{i}\right)\left|\nabla \mathbf{u}_{i}\right|^{2} \varphi_{i} \mathrm{~d} \mathbf{x} \mid \\
& \quad \leq\left.\int_{\Omega_{i}} \alpha_{i}\left(k_{i}^{n}\right)|| \nabla \mathbf{u}_{i}^{n+1}\right|^{2}-\left|\nabla \mathbf{u}_{i}\right|^{2}|| \varphi_{i}\left|\mathrm{~d} \mathbf{x}+\int_{\Omega_{i}}\right| \alpha_{i}\left(k_{i}^{n}\right)-\left.\alpha_{i}\left(k_{i}\right)|| \nabla \mathbf{u}_{i}\right|^{2}\left|\varphi_{i}\right| \mathrm{d} \mathbf{x},
\end{aligned}
$$

thanks to Hypothesis 3.1, the Sobolev embedding from $\mathbf{H}^{1}\left(\Omega_{i}\right)$ to $L^{6}\left(\Omega_{i}\right)$ and Hölder inequality, we can write for all $\varphi_{i} \in \mathcal{D}\left(\Omega_{i}\right)$,

$$
\begin{aligned}
& \left.\left|\int_{\Omega_{i}} \alpha_{i}\left(k_{i}^{n}\right)\right| \nabla \mathbf{u}_{i}^{n+1}\right|^{2}-\alpha_{i}\left(k_{i}\right)\left|\nabla \mathbf{u}_{i}\right|^{2} \varphi_{i} \mathrm{~d} \mathbf{x} \mid \\
& \quad \leq \delta_{1}\left\|\varphi_{i}\right\|_{\infty}\left\|\nabla \mathbf{u}_{i}^{n+1}\right\|_{L^{2}\left(\Omega_{i}\right)^{d}}\left\|\nabla \mathbf{u}_{i}\right\|_{L^{2}\left(\Omega_{i}\right)^{d}}\left\|\nabla\left(\mathbf{u}_{i}^{n+1}-\mathbf{u}_{i}\right)\right\|_{L^{2}\left(\Omega_{i}\right)^{d}}+c M^{2} \delta_{2}\left\|\varphi_{i}\right\|_{\infty}\left\|k_{i}^{n}-k_{i}\right\|_{H^{1}\left(\Omega_{i}\right)},
\end{aligned}
$$

where $c$ is a positive constant, depending only on the $\Omega_{i}$. Due to the strong convergence in $\mathbf{H}^{1}$ of the sequences $\left(\mathbf{u}_{i}^{n}\right)_{n}$ and $\left(k_{i}^{n}\right)_{n}$ to $\mathbf{u}_{i}$ and $k_{i}$

$$
\left|\int_{\Omega_{i}}\left(\alpha_{i}\left(k_{i}^{n}\right)\left|\nabla \mathbf{u}_{i}^{n+1}\right|^{2}-\alpha_{i}\left(k_{i}\right)\left|\nabla \mathbf{u}_{i}\right|^{2}\right) \varphi_{i} \mathrm{~d} \mathbf{x}\right| \leq \frac{\eta}{3} .
$$


Let now $\varphi_{i} \in W_{0}^{1, r}\left(\Omega_{i}\right)$. The density of $\mathcal{D}\left(\Omega_{i}\right)$ in $W_{0}^{1, r}\left(\Omega_{i}\right)$, implies that there exists a sequence $\left(\varphi_{i}^{m}\right)_{m}$ in $\mathcal{D}\left(\Omega_{i}\right)$, such that

$$
\left\|\varphi_{i}^{m}-\varphi_{i}\right\|_{L^{3}\left(\Omega_{i}\right)} \leq c\left(\Omega_{i}\right)\left\|\varphi_{i}^{m}-\varphi_{i}\right\|_{W^{1, r}\left(\Omega_{i}\right)} \leq \frac{\eta}{3},
$$

where $c\left(\Omega_{i}\right)$ only depends on $\Omega_{i}$. Finally, using the triangular inequality, we obtain

$$
\begin{aligned}
\left|\int_{\Omega_{i}}\left(\alpha_{i}\left(k_{i}^{n}\right)\left|\nabla \mathbf{u}_{i}^{n+1}\right|^{2}-\alpha_{i}\left(k_{i}\right)\left|\nabla \mathbf{u}_{i}\right|^{2}\right) \varphi_{i} \mathrm{~d} \mathbf{x}\right| & \left|\int_{\Omega_{i}}\left(\alpha_{i}\left(k_{i}^{n}\right)\left|\nabla \mathbf{u}_{i}^{n+1}\right|^{2}-\alpha_{i}\left(k_{i}\right)\left|\nabla \mathbf{u}_{i}\right|^{2}\right) \varphi_{i}^{m} \mathrm{~d} \mathbf{x}\right| \\
& +\left.\left|\int_{\Omega_{i}} \alpha_{i}\left(k_{i}^{n}\right)\right| \nabla \mathbf{u}_{i}^{n+1}\right|^{2}\left(\varphi_{i}^{m}-\varphi_{i}\right) \mathrm{d} \mathbf{x}|+| \int_{\Omega_{i}} \alpha_{i}\left(k_{i}\right)\left|\nabla \mathbf{u}_{i}\right|^{2}\left(\varphi_{i}^{m}-\varphi_{i}\right) \mathrm{d} \mathbf{x} \mid .
\end{aligned}
$$

Thus,

$$
\begin{aligned}
\left|\int_{\Omega_{i}}\left(\alpha_{i}\left(k_{i}^{n}\right)\left|\nabla \mathbf{u}_{i}^{n+1}\right|^{2}-\alpha_{i}\left(k_{i}\right)\left|\nabla \mathbf{u}_{i}\right|^{2}\right) \varphi_{i} \mathrm{~d} \mathbf{x}\right| \leq & \left\|\alpha_{i}\right\|_{\infty}\left\|\nabla \mathbf{u}_{i}^{n+1}\right\|_{L^{3}\left(\Omega_{i}\right)^{d}}^{2}\left\|\varphi_{i}^{m}-\varphi_{i}\right\|_{L^{3}\left(\Omega_{i}\right)} \\
& +\frac{\varepsilon}{3}+\left\|\alpha_{i}\right\|_{\infty}\left\|\nabla \mathbf{u}_{i}\right\|_{L^{3}\left(\Omega_{i}\right)^{d}}^{2}\left\|\varphi_{i}^{m}-\varphi_{i}\right\|_{L^{3}\left(\Omega_{i}\right)} .
\end{aligned}
$$

We deduce that $\forall \varphi_{i} \in W_{0}^{1, r}\left(\Omega_{i}\right), \lim _{n \rightarrow \infty} \int_{\Omega_{i}} \alpha_{i}\left(k_{i}^{n}\right)\left|\nabla \mathbf{u}_{i}^{n+1}\right|^{2} \varphi_{i} \mathrm{~d} \mathbf{x}=\int_{\Omega_{i}} \alpha_{i}\left(k_{i}\right)\left|\nabla \mathbf{u}_{i}\right|^{2} \varphi_{i} \mathrm{~d} \mathbf{x}$.

\section{Third step. Boundary conditions of TKE on $\Gamma$}

In this step, we show that $k_{i}=\lambda\left|\mathbf{u}_{1}-\mathbf{u}_{2}\right|^{2}$ on $\Gamma$.

Consider the following triangular inequality

$$
\left\|\lambda\left|\mathbf{u}_{1}-\mathbf{u}_{2}\right|^{2}-k_{i}\right\|_{H^{\frac{1}{2}(\Gamma)}} \leq\left\|\lambda\left|\mathbf{u}_{1}^{n}-\mathbf{u}_{2}^{n}\right|^{2}-k_{i}\right\|_{H^{\frac{1}{2}(\Gamma)}}+\left\|\lambda\left|\mathbf{u}_{1}^{n}-\mathbf{u}_{2}^{n}\right|^{2}-\lambda\left|\mathbf{u}_{1}-\mathbf{u}_{2}\right|^{2}\right\|_{H^{\frac{1}{2}(\Gamma)}} \cdot
$$

Due to the strong convergence of $\left(k_{i}^{n}\right)_{n}$ to $k_{i}$ in $H^{1}\left(\Omega_{i}\right)$, and thanks to the continuity of the trace operator from $H^{1}\left(\Omega_{i}\right)$ to $H^{\frac{1}{2}}(\Gamma)$, the sequence $k_{i}^{n}$ converges strongly to $k_{i}$ in $H^{\frac{1}{2}}(\Gamma)$. Furthermore, $k_{i}^{n}=\lambda\left|\mathbf{u}_{1}^{n+1}-\mathbf{u}_{2}^{n+1}\right|^{2}$ on $\Gamma$. Thus,

strongly in $H^{\frac{1}{2}}(\Gamma)$.

$$
\lim _{n \rightarrow \infty} \lambda\left|\mathbf{u}_{1}^{n+1}-\mathbf{u}_{2}^{n+1}\right|^{2}=k_{i},
$$

Let us now prove that

$$
\lim _{n \rightarrow \infty}|| \lambda\left|\mathbf{u}_{1}^{n}-\mathbf{u}_{2}^{n}\right|^{2}-\lambda\left|\mathbf{u}_{1}-\mathbf{u}_{2}\right|^{2} \|_{H^{\frac{1}{2}(\Gamma)}}=0 .
$$

Using the identity $a^{2}-b^{2}=(a-b)(a+b)$ and Lemma 3.5 , we obtain

$$
\left\|\lambda\left|\mathbf{u}_{1}^{n}-\mathbf{u}_{2}^{n}\right|^{2}-\lambda\left|\mathbf{u}_{1}-\mathbf{u}_{2}\right|^{2}\right\|_{H^{\frac{1}{2}}(\Gamma)} \leq \lambda\left\|\left(\mathbf{u}_{1}^{n}-\mathbf{u}_{1}\right)-\left(\mathbf{u}_{2}^{n}-\mathbf{u}_{2}\right)\right\|_{\mathbf{H}^{\frac{1}{2}(\Gamma)}}\left\|\left(\mathbf{u}_{1}^{n}+\mathbf{u}_{1}\right)-\left(\mathbf{u}_{2}^{n}+\mathbf{u}_{2}\right)\right\|_{W^{1-\frac{1}{3+\varepsilon}, 3+\varepsilon}(\Gamma)^{d}},
$$

so,

$$
\begin{aligned}
\| \lambda\left|\mathbf{u}_{1}^{n}-\mathbf{u}_{2}^{n}\right|^{2} & -\lambda\left|\mathbf{u}_{1}-\mathbf{u}_{2}\right|^{2} \|_{H^{\frac{1}{2}(\Gamma)}} \\
& \leq \lambda\left[\left\|\mathbf{u}_{1}^{n}-\mathbf{u}_{1}\right\|_{\mathbf{H}^{\frac{1}{2}(\Gamma)}}+\left\|\mathbf{u}_{2}^{n}-\mathbf{u}_{2}\right\|_{\mathbf{H}^{\frac{1}{2}}(\Gamma)}\right]\left[\left\|\mathbf{u}_{1}^{n}+\mathbf{u}_{1}\right\|_{W^{1-\frac{1}{3+\varepsilon}, 3+\varepsilon}(\Gamma)^{d}}+\left\|\mathbf{u}_{2}^{n}+\mathbf{u}_{2}\right\|_{W^{1-\frac{1}{3+\varepsilon}, 3+\varepsilon}(\Gamma)^{d}}\right] .
\end{aligned}
$$

Due to the continuity of the trace operators from $W^{1,3+\varepsilon}\left(\Omega_{1}\right)^{d}$ to $W^{1-\frac{1}{3+\varepsilon}, 3+\varepsilon}(\Gamma)^{d}$ and from $\mathbf{H}^{1}\left(\Omega_{i}\right)$ to $\mathbf{H}^{\frac{1}{2}}(\Gamma)$, and by Hypothesis 3.1, there exists a positive constant $c$, that only depends on the domains $\Omega_{i}$, such that

$$
\left\|\lambda\left|\mathbf{u}_{1}^{n}-\mathbf{u}_{2}^{n}\right|^{2}-\lambda\left|\mathbf{u}_{1}-\mathbf{u}_{2}\right|^{2}\right\|_{H^{\frac{1}{2}}(\Gamma)} \leq c M \lambda\left(\left\|\mathbf{u}_{1}^{n}-\mathbf{u}_{1}\right\|_{\mathbf{H}^{1}\left(\Omega_{1}\right)}+\left\|\mathbf{u}_{2}^{n}-\mathbf{u}_{2}\right\|_{\mathbf{H}^{1}\left(\Omega_{2}\right)}\right) .
$$


Finally, due to the strong convergence of $\left(\mathbf{u}_{i}^{n}\right)_{n}$ to $\mathbf{u}_{i}$ in $\mathbf{H}^{1}\left(\Omega_{i}\right)$, we deduce that

$$
\lim _{n \rightarrow \infty}\left\|\lambda\left|\mathbf{u}_{1}^{n}-\mathbf{u}_{2}^{n}\right|^{2}-\lambda\left|\mathbf{u}_{1}-\mathbf{u}_{2}\right|^{2}\right\|_{H^{\frac{1}{2}(\Gamma)}}=0 .
$$

Consequently, $k_{i}=\lambda\left|\mathbf{u}_{1}-\mathbf{u}_{2}\right|^{2}$ on $\Gamma$. This, finishes the proof of this theorem.

\section{Numerical EXPERIMENTS}

To conclude this paper, we use the algorithm introduced to solve the interaction of the ocean and the atmosphere in a simplified geometry. The discretization is performed using a Spectral method based on Legendre polynomials (see $[2,10]$ or $[11]$ for instance) that we have implemented in FreeFEM3D".

The algorithm presented in this paper is nonlinear, thus it cannot be used "as is". Various strategies could be employed to treat this nonlinearity (Newton or fixed point algorithms, etc.). According to the monotonic nature of the nonlinear friction boundary condition on the boundary $\Gamma$, we choose to linearize the term

$$
\int_{\Gamma}\left|\mathbf{u}_{i}^{n+1}-\mathbf{u}_{j}^{n+1}\right|\left(\mathbf{u}_{i}^{n+1}-\mathbf{u}_{j}^{n+1}\right) \cdot \mathbf{v}_{i} \mathrm{~d} \tau
$$

of Problem 1. We replace it by

$$
\int_{\Gamma}\left|\mathbf{u}_{i}^{n}-\mathbf{u}_{j}^{n}\right|\left(\mathbf{u}_{i}^{n+1}-\mathbf{u}_{j}^{n+1}\right) \cdot \mathbf{v}_{i} \mathrm{~d} \tau .
$$

This term is linear and still monotonic in the unknowns $\left(\mathbf{u}_{1}^{n+1}, \mathbf{u}_{2}^{n+1}\right)$, due to property (3.4). The problems for $\left(\mathbf{u}_{1}^{n+1}, p_{1}^{n+1}\right)$ and $\left(\mathbf{u}_{2}^{n+1}, p_{2}^{n+1}\right)$ still are coupled but the overall problem is linear and admits a unique solution:

Obtain $\left(\mathbf{u}_{i}^{n+1}, p_{i}^{n+1}, k_{i}^{n+1}\right) \in \mathbf{X}_{i} \times L^{2}\left(\Omega_{i}\right) \times W^{1, r^{\prime}}\left(\Omega_{i}\right)$, such that $\forall\left(\mathbf{v}_{i}, q_{i}, \varphi_{i}\right) \in \mathbf{X}_{i} \times L^{2}\left(\Omega_{i}\right) \times W_{0}^{1, r}\left(\Omega_{i}\right)$,

$$
\begin{aligned}
a_{i}\left(k_{i}^{n} ; \mathbf{u}_{i}^{n+1}, \nabla \mathbf{v}_{i}\right)+b_{i}\left(\mathbf{v}_{i}, p_{i}^{n+1}\right)+\kappa_{i} \int_{\Gamma}\left|\mathbf{u}_{i}^{n}-\mathbf{u}_{j}^{n}\right|\left(\mathbf{u}_{i}^{n+1}-\mathbf{u}_{j}^{n+1}\right) \cdot \mathbf{v}_{i} \mathrm{~d} \tau & =\int_{\Omega_{i}} \mathbf{f}_{i} \cdot \mathbf{v}_{i} \mathrm{~d} \tau \\
\text { and } \quad b_{i}\left(\mathbf{u}_{i}^{n+1}, q_{i}\right) & =0 .
\end{aligned}
$$

To perform a somewhat realistic computation, we consider turbulent viscosities $\alpha_{i}$ and $\gamma_{i}$ with the structure $\nu_{t}+\ell \sqrt{k}$. We consider the data reported in [5]:

- Geometry:

$\left.-\Omega_{1}=\right] 0,5[\times] 0,1[\times] 0,1[$ describes the atmosphere;

$\left.-\Omega_{1}=\right] 0,5[\times] 0,-1[\times] 0,1[$ is the ocean.

- Physical data (taken from [5]):

$-\gamma_{1}\left(k_{1}\right)=3 \times 10^{-3}+0.277 \times 10^{-4} \sqrt{k_{1}} ;$

$-\gamma_{2}\left(k_{2}\right)=3 \times 10^{-2}+0.185 \times 10^{-5} \sqrt{k_{2}}$

$-\alpha_{i}(\cdot)=\gamma_{i}(\cdot)$, for $i=1,2$.

- Friction coefficients (coming also from [5]):

$-\kappa_{i}=10^{-3}$, for $i=1,2$; and

$-\lambda=5 \times 10^{-2}$.

These data correspond to an air-sea flow, each modeled by a simplified TKE-mixing layer turbulence model. The mixing lengths are calculated by wall laws. The friction coefficients, in their turn, are just tentative. The physical units are in MKS system. The velocity boundary conditions imposes that $\mathbf{u}_{1}=\mathbf{0}$ on $\Gamma_{1} \backslash \tilde{\Gamma}_{1}$, $\mathbf{u}_{1}=(1,0,0)$ on $\tilde{\Gamma}_{1}$ and $\mathbf{u}_{2}=\mathbf{0}$ on $\Gamma_{2}$, where $\tilde{\Gamma}_{1}$ is the upper face $(y=1)$ of $\Omega_{1}$. These settings are chosen in order to create a driven cavity-like flow in $\Omega_{1}$. One expects to generate another driven cavity-like flow in $\Omega_{2}$ rotating in the opposite sense.

\footnotetext{
$4_{\text {http: //www. freefem.org/ff } 3 d / \text {. }}$
} 


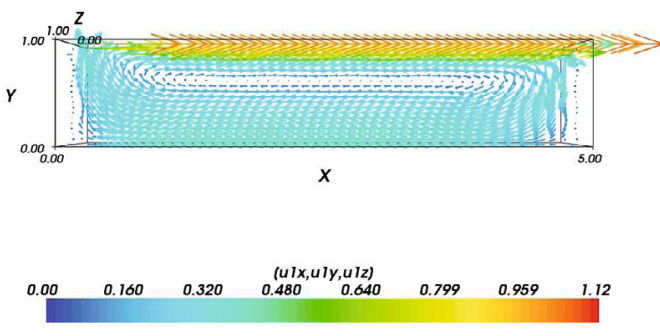

(a) $\mathbf{u}_{1}$ on the plane $z=0.5$

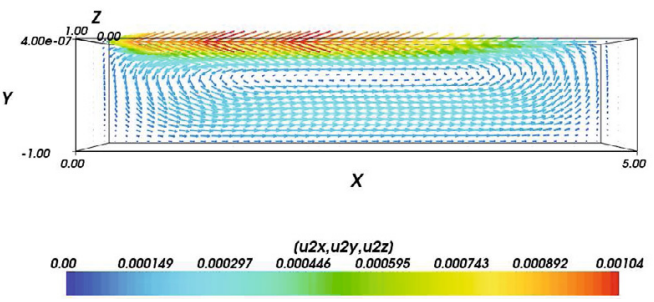

(c) $\mathbf{u}_{2}$ on the plane $z=0.5$

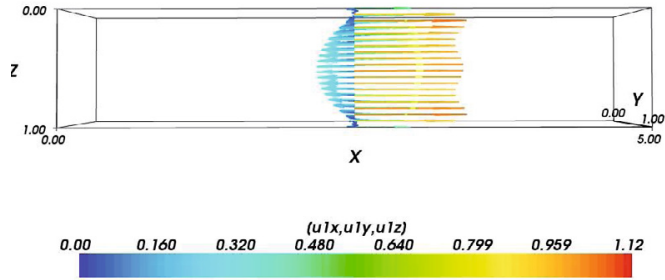

(b) $\mathbf{u}_{1}$ on the plane $x=2.5$

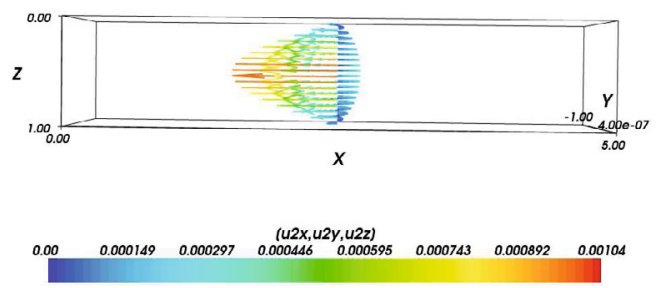

(d) $\mathbf{u}_{2}$ on the plane $x=2.5$

FIGURE 1. Velocity fields on cutting planes.

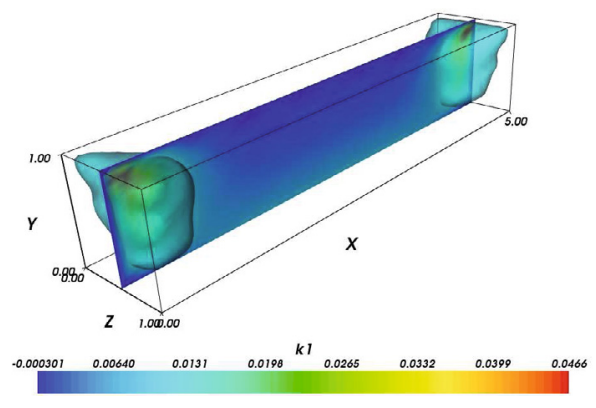

(a) $k_{1}$ on the plane $z=0.5$

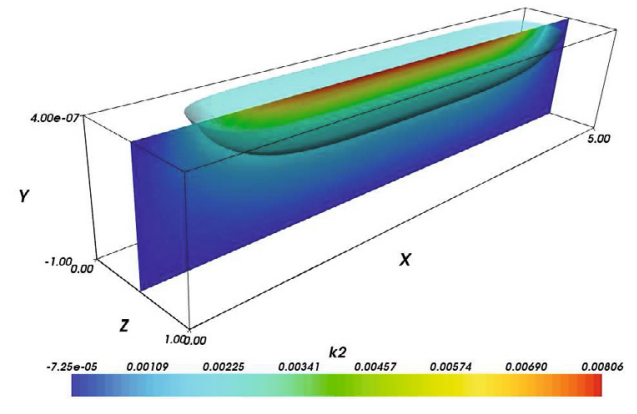

(b) $k_{2}$ on the plane $z=0.5$

Figure 2. Turbulent Kinetic Energy on cutting plane.

The results are obtained using a $\left(\mathbb{P}_{N}\right)^{3} \times \mathbb{P}_{N-2}$ discretization of $\left(\mathbf{u}_{\mathbf{i}}, p_{i}\right)$ to avoid spurious modes of the Stokes problem. The TKE is discretized using a $\mathbb{P}_{N}$ space. For this particular simulation we have chosen the following degrees for $\mathbf{u}_{i}$ and $k_{i}$ : 28 in direction $x$ and 8 in directions $y$ and $z$.

Computed velocity fields $\mathbf{u}_{\mathbf{i}}$ and the TKE $k_{i}$ are represented in Figures 1 and 2 . The results are quantitatively correct: The atmosphere flow generates a driven-lid like flow in the ocean, due to the boundary conditions on the velocity at $z=0$. Also, there is generation of TKE at $z=0$, again due to the TKE generation boundary conditions. 


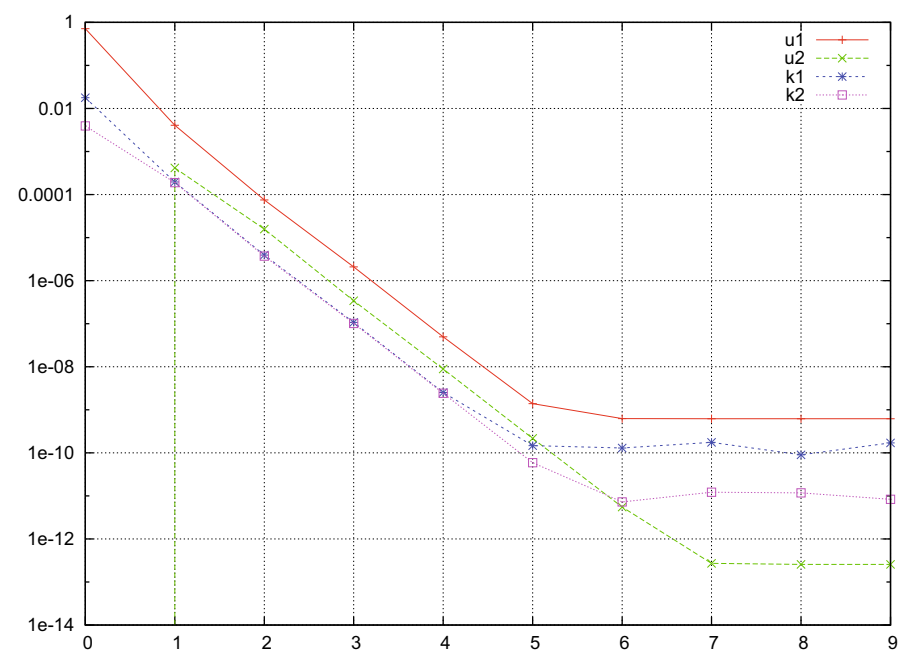

Figure 3. Convergence history: computed $L^{2}$ norm of the difference of successive iterates.

Figure 3 shows the expected exponential convergence rate of the algorithm due to its contractiveness. Note that this does not depend on the type of discretization (see [24] where Finite Element approximations have also been used).

\section{Conclusion}

In this paper, we have presented and analyzed a numerical scheme for the approximation of a model of two steady turbulent fluids with coupling at the interface. This is a simplified model for the atmosphere-ocean interaction, where we have neglected Coriolis forces and buoyancy effects, but have kept several non-linear interactions across the common boundary.

The proposed scheme is mainly linear, a monotone nonlinearity being just kept at the interface between the fluids. We showed the convergence of the triple $\left(\mathbf{u}_{i}^{n}, p_{i}^{n}, k_{i}^{n}\right)$, for reasonable hypothesis on the regularity of the velocity and the turbulent kinetic energy. This contribution ends with some numerical results, in good agreement with the theoretical expectations. Notably, the exponential convergence that appeared through the contractiveness of the sequences $\left(\mathbf{u}_{i}^{n}\right)_{n}$ and $\left(k_{i}^{n}\right)_{n}$ is found in our tests.

Several extensions to this work can be considered. To name a few, taking into consideration anisotropic diffusion and Coriolis forces (more realistic) are straightforward generalizations of the present analysis. Also, switching to unsteady incompressible flows should be possible using our approach. Taking into account buoyancy effects is more technically involved, but it should also be possible, similarly to the extension of the standard analysis for incompressible Navier-Stokes to buoyancy effects.

Acknowledgements. The authors are grateful to Christine Bernardi for her help and encouragements.

\section{REFERENCES}

[1] J.J.F. Adams and R.A. Fournier, Sobolev spaces. Second edition, Pure and Applied Mathematics Series, Elsevier/Academic Press (2003).

[2] C. Bernardi and Y. Maday, Approximations spectrales de problèmes aux limites elliptiques, Mathematics 83 Applications 10. Springer-Verlag (1992).

[3] C. Bernardi, T. Chacón Rebollo, R. Lewandowski and F. Murat, A model for two coupled turbulent fluids. I. Analysis of the system, in Nonlinear partial differential equations and their applications, Collège de France Seminar, Vol. XIV (Paris, 1997/1998), Stud. Math. Appl. 31, Amsterdam, North-Holland (2002) 69-102. 
[4] C. Bernardi, T. Chacón Rebollo, R. Lewandowski and F. Murat, A model for two coupled turbulent fluids. II. Numerical analysis of a spectral discretization. SIAM J. Numer. Anal. 40 (2003) 2368-2394.

[5] C. Bernardi, T. Chacón Rebollo, M. Gómez Mármol, R. Lewandowski and F. Murat, A model for two coupled turbulent fluids. III. Numerical approximation by finite elements. Numer. Math. 98 (2004) 33-66.

[6] C. Bernardi, T. Chacón Rebollo, F. Hecht and R. Lewandowski, Automatic insertion of a turbulence model in the finite element discretization of the Navier-Stokes Equations. Math. Mod. Meth. Appl. Sci. 19 (2009) 1139-1183.

[7] H. Brezis, Analyse Fonctionnelle: Théorie et Applications. Collection "Mathématiques Appliquées pour la Maîtrise", Masson (1983).

[8] F. Brossier and R. Lewandowski, Impact of the variations of the mixing length in a first order turbulent closure system. ESAIM: M2AN 36 (2002) 345-372.

[9] K. Bryan, A numerical method for the study of the circulation of the world ocean. J. Comput. Phys. 4 (1969) 347-369.

[10] C. Canuto, M.Y. Hussaini, A. Quarteroni and T.A. Zang, Spectral methods - Fundamentals in single domains. Springer, Berlin, Germany (2006).

[11] C. Canuto, M.Y. Hussaini, A. Quarteroni and T.A. Zang, Spectral methods - Evolution to complex geometries and applications to fluid dynamics. Springer, Berlin, Germany (2007).

[12] S. Del Pino and O. Pironneau, A fictitious domain based on general pde's solvers, in Proc. ECCOMAS 2001, Swansea, K. Morgan Ed., Wiley (2002).

[13] V. Girault and P.-A. Raviart, Finite Element Methods for Navier-Stokes Equations, Theory and Algorithms. Springer-Verlag, Germany (1986).

[14] P. Grisvard, Elliptic Problems in Nonsmooth Domains, Monographs and Studies in Mathematics 24. Pitman (Advanced Publishing Program), Boston, USA (1985).

[15] E. Hebey, Nonlinear analysis on manifolds: Sobolev spaces and inequalities, Courant Lecture Notes 5. American Mathematical Society, USA (1999).

[16] B.E. Launder and D.B. Spalding, Mathematical Modeling of Turbulence. Academic Press, London, UK (1972).

[17] J. Lederer and R. Lewandowski, A RANS 3D model with unbounded eddy viscosities. Ann. Inst. H. Poincaré Anal. Non Linéaire 24 (2007) 413-441.

[18] R. Lewandowski, Analyse Mathématique et Océanographie. Collection Recherches en Mathématiques Appliquées, Masson (1997).

[19] R. Lewandowski, The mathematical analysis of the coupling of a turbulent kinetic energy equation to the Navier-Stokes equation with an eddy viscosity. Nonlinear Anal. 28 (1997) 393-417.

[20] J.-L. Lions and E. Magenes, Problèmes aux limites non homogènes et applications 3, Travaux et Recherches Mathématiques 20. Dunod, Paris, France (1970).

[21] B. Mohammadi and O. Pironneau, Analysis of the k-epsilon turbulence model. RAM: Research in Applied Mathematics. Masson, Paris (1994).

[22] J. Piquet, Turbulent Flows, Models and Physics. Springer, Germany (1999).

[23] D.C. Wilcox, Turbulence Modeling for CFD. Sixth edition, DCW Industries, inc. California, USA (2006).

[24] D. Yakoubi, Analyse et mise en oeuvre de nouveaux algorithmes en méthodes spectrales. Ph.D. Thesis, Université Pierre et Marie Curie, Paris, France (2007). 